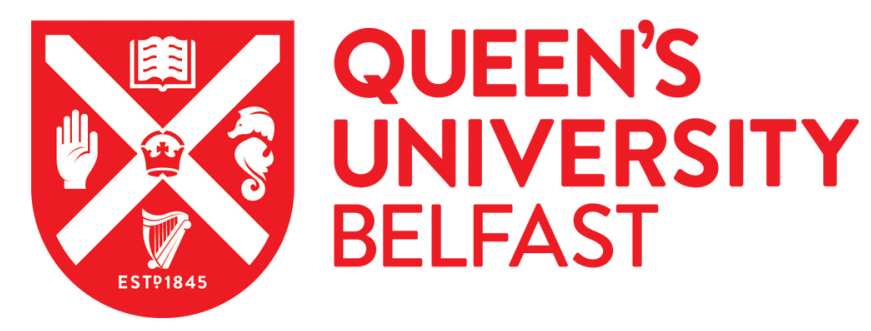

\title{
On The SEP Analysis of OFDM Index Modulation with Hybrid Low Complexity Greedy Detection and Diversity Reception
}

Crawford, J., Chatziantoniou, E., \& Ko, Y. (2017). On The SEP Analysis of OFDM Index Modulation with Hybrid Low Complexity Greedy Detection and Diversity Reception. IEEE Transactions on Vehicular Technology, 66(9), 8103-8118. https://doi.org/10.1109/TVT.2017.2691778

Published in:

IEEE Transactions on Vehicular Technology

Document Version:

Peer reviewed version

Queen's University Belfast - Research Portal:

Link to publication record in Queen's University Belfast Research Portal

Publisher rights

(c) 2017 IEEE.

This work is made available online in accordance with the publisher's policies. Please refer to any applicable terms of use of the publisher.

\section{General rights}

Copyright for the publications made accessible via the Queen's University Belfast Research Portal is retained by the author(s) and / or other copyright owners and it is a condition of accessing these publications that users recognise and abide by the legal requirements associated with these rights.

Take down policy

The Research Portal is Queen's institutional repository that provides access to Queen's research output. Every effort has been made to ensure that content in the Research Portal does not infringe any person's rights, or applicable UK laws. If you discover content in the Research Portal that you believe breaches copyright or violates any law, please contact openaccess@qub.ac.uk. 


\title{
On The SEP Analysis of OFDM Index Modulation with Hybrid Low Complexity Greedy Detection and Diversity Reception
}

\author{
James Crawford, Student Member, IEEE, Eleftherios Chatziantoniou, Member, IEEE, \\ and Youngwook Ko, Member, IEEE
}

\begin{abstract}
In this work, we investigate the performance of multicarrier index keying (MCIK) orthogonal frequency division multiplexing (OFDM) proposing a hybrid low complexity detection and diversity reception. For performance analysis, we derive novel exact closed-form expressions for the average pairwise error probability (PEP) and symbol error probability (SEP) of three detection methods: a greedy detector (GD), and a GD with maximal ratio combining (MRC) or selection combining (SC). Approximate and accurate expressions for the average PEP and SEP are also analyzed in closed-form. The derived expressions provide a useful insight into the error performance of MCIKOFDM with the hybrid detector in low, moderate and extreme rate of sparse sub-carrier indices activation. The effects of multiple antennas and sparse sub-carriers activation on the SEP are addressed in several extreme cases; decreasing the average SEP exponentially with proper choice of both sub-carrier activation rate and number of antennas. In comparison with the benchmark detection, the numerical results and simulations clearly show that the proposed schemes can benefit from diversity gain, at substantially reduced complexity. The derived SEP expressions and analyses will be useful to evaluate various concepts of MCIK OFDM in low-power device applications.
\end{abstract}

Index Terms-Greedy detection (GD), multicarrier index keying (MCIK), orthogonal frequency division multiplexing (OFDM), symbol error probability (SEP).

\section{INTRODUCTION}

Multicarrier modulation schemes have become increasingly popular due to their characteristically high data-rates. In particular, orthogonal frequency division multiplexing (OFDM) has been used in many wireless standards such as IEEE 802.11, IEEE 802.16, and 3GPP's long term evolution (LTE), due to its robustness to inter-symbol-interference (ISI) that occurs over frequency selective fading channels.

Recently, the concept of multicarrier index keying (MCIK) was integrated with OFDM in [1] and [2]. In particular, the MCIK intends to utilize the indices of sub-carriers as an additional dimension to the conventional two dimensional M-ary constellation symbols. In the MCIK, the use of subcarrier indices as an additional degree of freedom facilitates the transmission of additional data without increasing the power consumption or bandwidth. Moreover, provided that

This work was presented in part at the IEEE Personal, Indoor, Mobile Radio Communications, August 2015. The authors are with the Electronics, Communications and Information Technology Institute, Queen's University Belfast, Belfast BT3 9DT United Kingdom (e-mail: \{jcrawford23, 1.chatziantoniou, y.ko\}@qub.ac.uk).
MCIK-OFDM, also known as OFDM with index modulation (OFDM-IM), only activates a subset of sub-carriers, the complexity of transceiver structure is reduced as less modulators/demodulators are required. Hence, MCIK-OFDM is a low-cost, low-complexity modulation technique, which is ideal for future high data-rate device-to-device (D2D) wireless systems.

The MCIK concept was initially inspired by spatial modulation (SM) [3], and generalized SM (GSM) [4], which were introduced as multi-antenna transmission schemes where data is not only transmitted via data symbols but also via the indices of the transmitting antenna. Similarly, code index modulation (CIM) [5], and generalized CIM [6], were also inspired by the SM concept. CIM is a transmission scheme that uses multiple spreading codes, where a certain code is selected and its index is used as a mechanism to carry data. In [7] it is shown that CIM outperforms SM in terms of energy efficiency, spectral efficiency, complexity, and error performance, while being simpler to implement in terms of hardware complexity. The principle of MCIK, SM, and CIM comes from exploiting the indices of an additional dimension over which the data is delivered. A hybrid single-carrier OFDM and SM (SC-SM) transmission scheme is proposed in [8] as a promising candidate for large scale multi-input multi-output (LS-MIMO) aided multi-user (MU) communication systems. This study includes complex channel scenarios suitable for D2D communications. This novel low-complexity, and low-cost scheme is capable of adopting low-complexity single-stream based detection, while only requiring a single $\mathrm{RF}$ chain.

The popularity of MCIK has increased significantly in recent years. In [9], it has been shown that MCIK-OFDM achieves a balanced trade-off between error performance and spectral efficiency. In [10], a tight closed-form approximation of the bit error rate is derived. In [11] interleaving was introduced to improve the error performance in low signal-to-noise power ratio (SNR) regions by increasing the Euclidean distance between recovered M-ary symbols. This work was then extended to vehicular communications in [12]. An MCIK variant is proposed in [13], which shows that transmission rates for cell-edge users in a proposed OFDMA network can be significantly improved. The optimal number of active sub-carriers is investigated in [14] and [15]. The diversity gain of OFDM-IM is improved with the use of coordinate interleaving in [16]. In [17] the spectral efficiency was improved with a generalized index modulation scheme for 
MCIK-OFDM, where the number of active sub-carriers is no longer fixed. A low complexity compressive sensing technique for an sparsely modulated MCIK variant is introduced in [18]. The performance of a sparse MCIK is also analyzed over highly correlated sub-carriers in [19]. The ergodic achievable rate of MCIK-OFDM techniques is investigated in [20]. An MCIK scheme that utilizes all possible active sub-carrier index combinations is proposed in [21]. The exact coding gain of an MCIK-OFDM technique is derived in [22]. More recently, MCIK-OFDM in a multiple-input multiple-output (MIMO) structure is investigated with the maximum likelihood (ML) detector in [23].

Since its development, the main focus of the aforementioned studies is the improvement of the error performance, diversity gain and spectral efficiency. For a successful development of MCIK-OFDM D2D systems, complexity is critical. To tackle this issue, [24] recently proposed a low complexity energy based detection scheme for MCIK-OFDM and evaluated the pairwise error probability. In [25], an insight on the error performance of MCIK-OFDM within enclosed metallic structures, such as in-vehicular D2D wireless networks was provided. However, the achievable power gain of such energy based detection is inferior to maximum likelihood (ML) detection, which is traded-off for low complexity. It is timely to investigate methods for improving the error performance simultaneously at reduced complexity, and potential of MCIKOFDM which utilizes energy based detection and multiple antennas has been overlooked in this field.

In this work, we consider a D2D communications system where a device intends to communicate with a central device, employing the MCIK-OFDM method. Allowing multiple antennas only at the central device, we investigate a new hybrid low complexity detection and diversity reception scheme at affordable complexity. Namely, the greedy detection (GD) scheme is proposed to detect MCIK-OFDM signals by exploiting jointly the energy detection and two different diversity reception techniques: maximal ratio combining (MRC) and selection combining (SC). We aim not only to increase the system reliability but also to reduce the detection complexity. Particularly, the proposed detection scheme develops the opportunity for providing synergy effects between energy detection of active sub-carrier indices and diversity reception of active sub-carrier signals. The main contributions can be summarized as follows:

- We create the structure of a hybrid GD and two different antenna combining techniques: GD-MRC and GD-SC. This structure employs different numbers of active subcarriers and receive antennas. We develop GD-based MCIK-OFDM algorithms with multiple receive antennas to improve the reliability at low complexity.

- For performance evaluation, we derive closed-form expressions for both the average pairwise error probability (PEP) and the average symbol error probability (SEP) of the MCIK-OFDM employing three detection approaches: GD, GD-MRC, and GD-SC.

- We generalize the error probability expressions with any number of active sub-carriers and receive antennas. To the best of our knowledge, the average SEP expression in closed form has not been discussed yet for MCIKOFDM with diversity reception. Using the expressions, we analyze the exact and approximate behaviour of error performance with respect to the number of active subcarriers and antennas. This allows for the potential of the proposed algorithms to be analytically addressed with various pragmatic setups.

- We provide theoretical guidelines of the performance of MCIK-OFDM with GD-MRC through asymptotic analysis of the average SEP. For this, we consider various extreme cases including low and extreme rate of subcarrier index activation.

- Computational complexity has been discussed to highlight the achieved reduction in the detection complexity . The proposed schemes can bring significant complexity reduction over the ML that suffers from a brute force search with very high complexity.

- The numerical and asymptotic results clearly show that the proposed schemes are superior to the existing MCIKOFDM and conventional OFDM methods, in terms of the reliability at affordable complexity.

This work is a continuation of the work presented in [24] and [26]. The difference of this paper from the previous works contains that the analytical expressions presented in this work have been extended to account for both the average SEP and the average PEP, unlike [24] and [26] which focused on the average PEP alone; that both the selection combining and the maximal ratio combining have been addressed, unlike [26]; that three extreme cases are considered to investigate the asymptotic behavior of the average SEP, with respect to the MCIK parameters and the sparsity ratio; and that a trade-off between performance and complexity is addressed, taking into account ML, LLR, and GD, as well as diversity techniques of both MCIK-OFDM and OFDM.

The rest of this paper is organized as follows. In Section II the system model of MCIK-OFDM with GD-based diversity reception is presented. In Section III closed-form expression for the exact and approximate average PEP for GD, GD-MRC and GD-SC are derived. Similarly, in Section IV closed-form expressions for the upper bounded and approximate average SEP for GD, GD-MRC and GD-SC are derived. Asymptotic analysis is performed in Section V, followed by complexity comparison in Section VI. In Section VII numerical and simulation results are presented. Finally, Section VIII concludes the presented work.

Annotation: Lower-case bold and upper-case bold letters are used for vectors and matrices, respectively. $\mathcal{S}$ denotes the complex signal constellation. $\lceil\cdot\rceil$ and $\lfloor\cdot\rfloor$ represent the ceiling and floor functions, respectively. $|\cdot|$ stands for the absolute value. $\|\cdot\|$ stands for the Euclidean norm. $\left(\begin{array}{l}n \\ k\end{array}\right)$ is the binomial coefficient for $n$ choose $k, \Theta(\cdot)$ is the Big-Theta notation, $\mathcal{O}(\cdot)$ is the Big-O notation.

\section{SyStem ModeL}

\section{A. MCIK-OFDM}

Consider a up-link single-input multiple-output (SIMO) system where a device with a single antenna intends to 


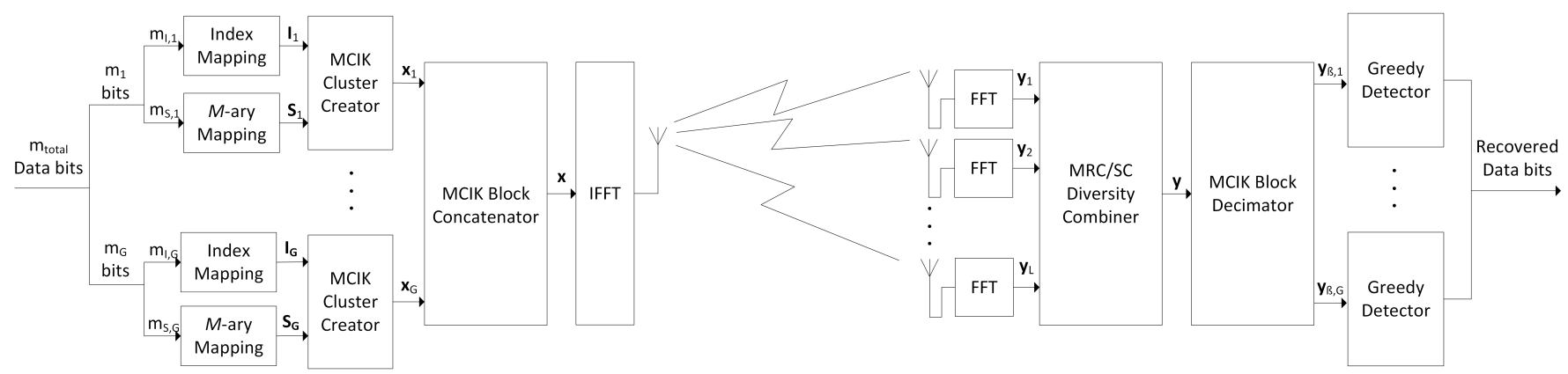

Fig. 1. MCIK-OFDM with a Joint Greedy Detector and Diversity Reception Block Diagram

communicate with a central device with $L$ antennas. An MCIK based OFDM method with a joint low complexity detector and antenna diversity reception, as seen in Fig. 1, is investigated. Suppose a transmitter has a single antenna while the receiver has $L$ antennas. The post OFDM system has $N_{c}=G N$ total sub-carriers, where there are $G$ clusters of $N$ sub-carriers. In a conventional OFDM system, a stream of M-QAM symbols is first serial-to-parallel converted, where every $N$ symbols per cluster are grouped into a vector $\mathbf{s}_{O F D M}=\left[s_{1}, s_{2}, \ldots, s_{N}\right]^{T}$ and each M-ary symbol $s_{i} \in \mathcal{S}$ is used to modulate each subcarrier.

Different from the conventional OFDM, only $K$ out of $N$ sub-carriers are dynamically activated according to incoming data bits. More specifically, in every transmission, only $K$ sub-carriers are modulated to deliver M-ary symbols, while the remaining $N-K$ sub-carriers are zero padded.

Let a set of $K$ active sub-carrier indices, be denoted by $\mathbf{I}_{g}=\left[i_{g}(1), \ldots, i_{g}(K)\right]$, for cluster $g$, where $i_{g}(k) \in\{(g-1) N+1, \ldots, g N\}, g=1, \ldots, G$, and $k=1, \ldots, K$. Accordingly, a cluster of Mary symbols is denoted by $\mathbf{s}_{g}=\left[s_{g}(1), \ldots, s_{g}(K)\right]$ where $s_{g}(k) \in \mathcal{S}$. Using both $\mathbf{I}_{g}$ and $\mathbf{s}_{g}$, the MCIK block for cluster $g$ is generated as $\mathbf{x}_{g}=$ $\left[x_{g}((g-1) N+1), x_{g}((g-1) N+2), \cdots, x_{g}(g N)\right]^{T}$, where $x_{g}(\alpha) \in \mathcal{S}$, for $\alpha \in \mathbf{I}_{g}$, and $x_{g}(\widetilde{\alpha})=0$, for $\widetilde{\alpha} \notin \mathbf{I}_{g}$. The transmit power of non-zero data symbols is $\mathbb{E}\left\{\left|x_{g}(\alpha)\right|^{2}\right\}=N / K \cdot E_{s}$, where $E_{s}$ is the average power per M-ary symbol and $\omega=N / K$ is the power allocation coefficient. The MCIK clusters are then concatenated together to create the MCIK block, i.e., $\mathbf{x}=\left[\mathbf{x}_{1}^{T}, \mathbf{x}_{2}^{T}, \cdots, \mathbf{x}_{G}^{T}\right]^{T}$, as shown in Fig. 1. Unlike the conventional OFDM, $x$ conveys the information not only via the $\mathrm{M}$-ary symbols but also through the indices of the activated sub-carriers that carry the symbols.

The total number of active sub-carrier index combinations is $\left(\begin{array}{l}N \\ K\end{array}\right)$. However, for simplicity and efficient mapping of the binary bits $C=2^{\left.\log _{2}\left(\begin{array}{c}N \\ K\end{array}\right)\right\rfloor}$ combinations are used. In each transmission per cluster, $m_{I, g}=\left\lfloor\log _{2}\left(\begin{array}{c}N \\ K\end{array}\right)\right\rfloor$ bits determine the combination of active sub-carriers. In addition, $m_{S, g}=$ $K \log _{2} M$ bits determine the M-ary symbols transmitted on the activated sub-carriers. Thus, the transmit data-rate (bits per cluster) can be given as,

$$
\bar{R}_{M C I K}=\left\lfloor\log _{2}\left(\begin{array}{l}
N \\
K
\end{array}\right)\right\rfloor+K \log _{2} M,
$$

with $m_{\text {Total }}=\sum_{g=1}^{G} m_{I, g}+m_{S, g}$ bits per transmit interval and $m_{g}=m_{I, g}+m_{S, g}$ bits in every cluster.

Suppose a frequency-selective Rayleigh fading SIMO channel. In particular the channel impulse response (CIR) in the frequency domain, between the transmitter and the $l^{\text {th }}$ receive antenna can be defined as $\mathbf{H}_{l}=\operatorname{diag}\left(h_{l}(1), \ldots, h_{l}\left(N_{c}\right)\right)$, where $l=1, \ldots, L$, and $h_{l}(\alpha)$ for $\alpha \in \mathbf{I}_{g}, \forall g$, represent Rayleigh fading channel as being independent and identically distributed (i.i.d.) complex Gaussian random variable (RV) with zero mean and unit variance, i.e., $h_{l}(\alpha) \sim \mathcal{C N}(0,1)$, and $h_{l}(\widetilde{\alpha})$ for $\widetilde{\alpha} \notin \mathbf{I}_{g}$ are zeros. The input-output model, for the $l^{\text {th }}$ antenna pair is given as,

$$
\mathbf{y}_{l}=\mathbf{H}_{l} \mathbf{x}+\mathbf{n}_{l},
$$

where $\mathbf{y}_{l}=\left[y_{l}(1), \ldots, y_{l}\left(N_{c}\right)\right]^{T}$ denotes the received MCIKOFDM signal block, $\mathrm{x}$ denotes the MCIK-OFDM signal block, and $\mathbf{n}_{l}=\left[n(1), \ldots, n\left(N_{c}\right)\right]^{T}$ is an independent additive white Gaussian noise (AWGN) vector on the $l^{\text {th }}$ diversity branch, where $n_{l}(j) \sim \mathcal{C N}\left(0, N_{0}\right)$, for $j=1, \ldots N_{c}$ and $N_{0}$ is the noise variance. Denoted by $\bar{\gamma}$, the average SNR per active sub-carrier is given as $\bar{\gamma}=\omega \cdot E_{s} / N_{0}$. Here, $\omega$ is related to sparsity ratio $(\tau=1 / \omega)$, which is ratio of the number of active sub-carriers to that of available sub-carriers.

As for the reception, the integration of the diversity combiner with the greedy detector, as shown in Fig. 1, is investigated. Particularly, after the fast Fourier transform (FFT), $L$ diversity branches, i.e., $\mathbf{y}_{1}, \mathbf{y}_{2}, \ldots, \mathbf{y}_{L}$, are combined using either maximal ratio combining (MRC) or selection combining (SC). In the following subsections the combined-output MCIK-OFDM block diversity reception is presented.

1) Maximal Ratio Combining for MCIK-OFDM: The output of the MRC can be rewritten as,

$$
\mathbf{y}_{M R C}=\mathbf{H}_{M R C} \mathbf{x}+\mathbf{n}_{M R C},
$$

where $\mathbf{H}_{M R C}=\mathbf{W}^{H} \overline{\mathbf{H}}$ is the effective channel matrix that results from the path combining, $\overline{\mathbf{H}}=\left[\mathbf{H}_{1}^{T}, \ldots, \mathbf{H}_{L}^{T}\right]^{T}$, $\mathbf{n}_{M R C}=\mathbf{W}^{H} \overline{\mathbf{n}}$ is the normalized AWGN vector at the combiner output, and $\overline{\mathbf{n}}=\left[\mathbf{n}_{1}^{T}, \ldots, \mathbf{n}_{L}^{T}\right]^{T}$ is a vector of the AWGN at each diversity branch. 


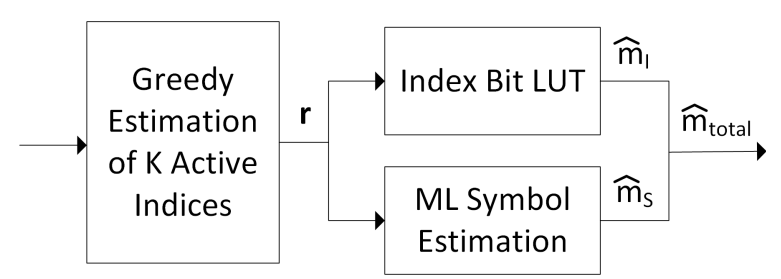

Fig. 2. Greedy Detector Block Diagram

2) Selection Combining for MCIK-OFDM: The output of the SC for the MCIK-OFDM can be written as,

$$
\mathbf{y}_{S C}=\mathbf{H}_{S C} \mathbf{x}+\mathbf{n}_{S C},
$$

where $\mathbf{H}_{S C}=\operatorname{diag}\left(h_{S C}(1), \ldots, h_{S C}\left(N_{c}\right)\right), h_{S C}(j)=$ $\max _{l}\left|h_{l}(j)\right|^{2}, \forall j$, and $\mathbf{n}_{S C}$ are the CIR and the AWGN vector of the diversity branch with the highest SNR, respectively.

For simplicity of notation, the combined-output signal can be generalized as,

$$
\mathbf{y}_{\beta}=\mathbf{H x}+\mathbf{n},
$$

where $\beta \in\{M R C, S C\}, \mathbf{H} \in\left\{\mathbf{H}_{M R C}, \mathbf{H}_{S C}\right\}$, and $\mathbf{n} \in$ $\left\{\mathbf{n}_{M R C}, \mathbf{n}_{S C}\right\}$, depending on which combining technique is used.

For given $\mathbf{y}_{\beta}$, the MCIK block decimator processes $\mathbf{y}_{\beta}=$ $\left[\mathbf{y}_{\beta, 1}^{T}, \mathbf{y}_{\beta, 2}^{T}, \cdots, \mathbf{y}_{\beta, G}^{T}\right]^{T}$, so that the combined-output signal vector, $\mathbf{y}_{\beta, g}=\mathbf{H}_{g} \mathbf{x}_{g}+\mathbf{n}_{g}$, is forwarded to the following detector, where $\mathbf{H}_{g}$, and $\mathbf{n}_{g}$ denote the post processing channel matrix, and the noise vector at cluster $g$, respectively. In the detector, both the M-ary symbols and the indices of the sub-carriers that carry them are recovered. For simplicity in analysis and without loss of generality, we address the detection process of only one cluster and the cluster index $g$ is omitted from subscripts hereafter as each cluster operates independently.

\section{B. Hybrid Greedy Detector and Diversity Reception}

A low-complexity detection scheme with diversity reception is proposed. The so-called GD needs a two stage detection process: the indices of the active sub-carriers and corresponding $\mathrm{M}$-ary symbols are estimated separately, as depicted in Fig. 2. In the first stage, GD measures the combined-output signal energy of each sub-carrier $\left|y_{\beta}(j)\right|^{2}$ and detects $K$ active sub-carriers with the greatest energy. Sub-carriers only under favorable channel fading are highly likely to be estimated as activate. In order to reduce the computational complexity in the detection of $K$ active sub-carriers, the first stage requires no channel information. In the second stage, the non-zero M-ary symbols in $\mathbf{x}$ are detected, applying the maximum likelihood (ML) decision individually to the $K$ estimated active subcarriers.

Detailed description of the GD process (i.e., stage 1 and stage 2) per cluster, is given.

Stage 1: Sense the $K$ greatest among $N$ received sub-carrier signal powers and detect the indices of the active sub-carriers.

1) Let a residual vector $\mathbf{z}=\mathbf{y}_{\beta}$. A demodulated vector $\mathbf{r}$ is set to a zero vector, i.e., $\mathbf{r}=\mathbf{0}_{1 \times N}$, and set the iteration count $t=0$.
TABLE I

EXAMPLE OF LUT FOR $N=4$ AND $K=2$

\begin{tabular}{|c|c|}
\hline $\mathbf{r}_{K}$ & $m_{I}$ bits \\
\hline \hline 1100 & 00 \\
\hline 1010 & 01 \\
\hline 1001 & 10 \\
\hline 0110 & 11 \\
\hline
\end{tabular}

2) Find the greatest element whose index $\hat{\alpha}$ is given by,

$$
\hat{\alpha}=\arg \max _{j}|z(j)|^{2},
$$

where $z(j)$ is the $j^{\text {th }}$ element of $\mathbf{z}$.

3) Let $r(\hat{\alpha})=z(\hat{\alpha})$ and $z(\hat{\alpha})=0$, and increment $t$ by $t=t+1$, where $r(\hat{\alpha})$ is the $\alpha^{\text {th }}$ element in $\mathbf{r}$.

4) Repeat stages 2 and 3 until $t=K$.

5) Set all non-zero elements in $\mathbf{r}$ equal to 1 . Recover the $m_{I}$ bits for $\mathbf{r}$ using an LUT. An example LUT for the case when $N=4$ and $K=2$ can be seen in Table I.

Stage 2: The M-ary symbols are estimated by using the ML criterion on sub-carriers of active indices $\hat{\alpha}$ as,

$$
\hat{x}(\hat{\alpha})=\arg \min _{x(\hat{\alpha}) \in \mathcal{S}}\left|y_{\beta}(\hat{\alpha})-h(\hat{\alpha}) x(\hat{\alpha})\right|^{2},
$$

where $h(\hat{\alpha})$ is the $\alpha^{\text {th }}$ diagonal element of $\mathbf{H}$ in (5), which is the equivalent channel matrix after the diversity reception.

Notice that the GD employs energy detection that significantly reduces the complexity in estimating a set of active subcarriers indices without the brute-force search of all possible index combinations. This leads to another complexity reduction in detecting M-ary symbols, which is separately done from the sub-carrier index detection.

\section{Pairwise Error Probability (PEP) Analysis}

\section{A. Exact Instantaneous PEP for GD with Diversity Reception}

Given that the GD uses the maximum received energy as a test statistic, the pairwise error event (PEE) is defined as the case when the greatest noise energy of any inactive subcarrier is greater than the energy of any active sub-carrier, i.e., $\max _{\widetilde{\alpha}}|n(\widetilde{\alpha})|^{2}>\|h(\alpha) x(\alpha)+n(\alpha)\|^{2}$, where $\alpha$ and $\tilde{\alpha}$ represent the indices of active and inactive sub-carriers, respectively. Hence the probability of the PEE occurring, i.e., the PEP, is given as,

$$
\begin{aligned}
P(\alpha \rightarrow \tilde{\alpha}) & =P\left(\max _{\widetilde{\alpha}}\left\{\left\|y_{\beta}(\widetilde{\alpha})\right\|\right\}^{2}>\left\|y_{\beta}(\alpha)\right\|^{2}\right) \\
& =P\left(\max _{\widetilde{\alpha}}\left\{\|n(\widetilde{\alpha})\|^{2}\right\}\right. \\
& \left.-\|h(\alpha) x(\alpha)+n(\alpha)\|^{2}>0\right) .
\end{aligned}
$$

The PEP can be defined in terms of the distribution of the power of the active and inactive sub-carriers, which is given as,

$$
\begin{aligned}
P(\alpha \rightarrow \tilde{\alpha}) & =\int_{0}^{\infty} \int_{\left|y_{\beta, \alpha}\right|^{2}}^{\infty} f_{\tilde{\alpha}}\left(\left|y_{\beta, \tilde{\alpha}}\right|^{2}\right) \\
& \times f_{\alpha}\left(\left|y_{\beta, \alpha}\right|^{2}\right) \mathrm{d}\left|y_{\beta, \tilde{\alpha}}\right|^{2} \mathrm{~d}\left|y_{\beta, \alpha}\right|^{2},
\end{aligned}
$$


where $f_{\tilde{\alpha}}\left(\left|y_{\beta, \tilde{\alpha}}\right|^{2}\right)$ is the probability density function (PDF) of the greatest power of inactive sub-carriers, $f_{\alpha}\left(\left|y_{\beta, \alpha}\right|^{2}\right)$ is the PDF of the power of an active sub-carrier, $\left|y_{\beta, \tilde{\alpha}}\right|^{2} \sim \chi_{2 f}^{2}$ is chi squared distributed with $2 f$ degrees of freedom, and with $\left|y_{\beta, \alpha}\right|^{2} \sim \chi_{2 f}^{2}\left(\gamma_{\beta, \alpha}\right)$ is non-central chi-square distributed with $2 f$ degrees of freedom and a non-centrality parameter, $\gamma_{\beta, \alpha} \in$ $\left\{\gamma_{M R C}(\alpha), \gamma_{S C}(\alpha)\right\}$, which is the instantaneous SNR on the $\alpha^{\text {th }}$ active sub-carrier index at the output of the MRC or SC.

The exact overall instantaneous PEP yields the PEP for $K>1$. It can be found using the law of total probability and $P(\alpha \rightarrow \tilde{\alpha})$, [26] and is expressed as;

$$
\begin{aligned}
i P E P & =\frac{K}{N} \sum_{\alpha=1}^{N} P(\alpha \rightarrow \tilde{\alpha}) \\
& =\frac{K}{N} \sum_{\alpha=1}^{N}\left[1-\sum_{q=0}^{N-K}\left(\begin{array}{c}
N-K \\
q
\end{array}\right) \frac{(-1)^{q}}{q+1} e^{\gamma_{\beta, \alpha}\left(\frac{1}{q+1}-1\right)}\right] .
\end{aligned}
$$

\section{B. Approximate Instantaneous PEP for GD with Diversity Reception}

The approximate overall instantaneous PEP can be found by applying Taylor series expansion to (10) and can be given by the weighted sum of simple exponentials as,

$$
i P E P_{\text {Approx. }}=\frac{K}{N} \sum_{\alpha=1}^{N}\left[\frac{N-K}{2} e^{\gamma_{\beta, \alpha}\left(-\frac{1}{2}\right)}\right] .
$$

Full details of the derivation can be found in [26].

\section{Average PEP for GD with MRC Reception (GD-MRC)}

For MRC diversity reception the distribution of the received SNR of the $\alpha^{\text {th }}$ sub-carrier is given as $\gamma_{M R C, \alpha} \sim \mathcal{X}_{2 L}^{2}$, where the mean $\mu=L \bar{\gamma}$, the variance $\sigma=2 L \bar{\gamma}$. For ease of notation $M R C$ is omitted from the subscript, hereafter. Since the SNR at the output of the combiner is the sum of $L$ diversity branch SNRs, the PDF of the SNR for MRC is given as,

$$
f_{\gamma}\left(\gamma_{\alpha}\right)=\frac{\gamma_{\alpha}^{L-1} e^{-\frac{\gamma_{\alpha}}{\bar{\gamma}}}}{\bar{\gamma}^{L}(L-1) !} .
$$

Using (10) and (12), the exact average PEP for MCIKOFDM GD-MRC can be formulated as,

$$
\overline{P E P}^{M R C}=\int_{0}^{\infty} i P E P\left(\gamma_{\alpha}\right) f_{\gamma}\left(\gamma_{\alpha}\right) d \gamma_{\alpha},
$$

The closed-form expression for the exact average PEP is given as,

$$
\overline{P E P}^{M R C}=K-K \sum_{q=0}^{N-K}\left(\begin{array}{c}
N-K \\
q
\end{array}\right) \frac{(-1)^{q}}{q+1}\left(\frac{q+1}{q \bar{\gamma}+q+1}\right)^{L} .
$$

Similarly, using (11) and (12), the close-form expression for the approximate average PEP is obtained,

$$
\overline{P E P}_{\text {Approx. }}^{M R C}=K \frac{N-K}{2}\left(\frac{2}{\bar{\gamma}+2}\right)^{L} .
$$

A detailed description of the derivations are shown in the Appendix A.
From (15), it can be shown that the achievable diversity order is $d=-\lim _{\bar{\gamma} \rightarrow \infty} \log \overline{P E P}_{A p \text { prox }}^{M R C} / \log \bar{\gamma}=L$. Note that (15) is clearly less complex than (14) as it no longer contains a summation and binomial coefficient terms. Also, it is worth pointing out from (15) that the average PEP behaves concavely with $K$ for given $N$ and $\bar{\gamma}$, due to $\partial^{2}\left[\overline{P E P}_{A p p r o x}^{M R C}\right] / \partial K^{2}<0, \forall K$. The strictly concave behaviour of (15) can easily produce the optimal $K$ of minimizing the approximate average PEP, as either the smallest or the largest over a given set of $K \mathrm{~s}$. For example, let $K \in\left\{K_{1}, \cdots, K_{m}\right\}$ be given for the required data-rate. Then, using (15), the optimal $K$ for minimizing the average PEP can be approximated to be $K_{\text {opt }}=\arg \min \left\{K_{1}\left(N-K_{1}\right), K_{m}\left(N-K_{m}\right)\right\}$.

\section{Average PEP for GD with SC Reception (GD-SC)}

For SC diversity reception the distribution of the received SNR of the $\alpha^{\text {th }}$ sub-carrier is given as $\gamma_{S C, \alpha} \sim \mathcal{X}_{2 L}^{2}$, where the mean $\mu=L \bar{\gamma}$, the variance $\sigma=2 L \bar{\gamma}$, and for ease of notation $S C$ is omitted from the subscript, hereafter. As the average SNR at the output of the combiner is the largest of $L$ such random variables, the PDF of the effective SNR for SC is given as,

$$
f_{\gamma}\left(\gamma_{\alpha}\right)=\frac{L}{\bar{\gamma}} e^{-\frac{\gamma_{\alpha}}{\bar{\gamma}}}\left[1-e^{-\frac{\gamma_{\alpha}}{\gamma}}\right]^{L-1} .
$$

After binomial expansion of $\left[1-e^{-\frac{\gamma_{\alpha}}{\gamma}}\right]^{L-1}$, (16) can be written as,

$$
f_{\gamma}\left(\gamma_{\alpha}\right)=\frac{L}{\bar{\gamma}} \sum_{l=0}^{L-1}\left(\begin{array}{c}
L-1 \\
l
\end{array}\right)(-1)^{l} e^{-\gamma_{\alpha} \frac{l+1}{\bar{\gamma}}} .
$$

Using (17), the average PEP for MCIK-OFDM GD-SC can be formulated as,

$$
\overline{P E P}^{S C}=\int_{0}^{\infty} i P E P\left(\gamma_{\alpha}\right) f_{\gamma}\left(\gamma_{\alpha}\right) d \gamma_{\alpha} .
$$

Averaging (10) and (11) over (17) yields the expressions for the exact and approximate average PEP for GD-SC, which are respectively given as,

$$
\begin{gathered}
\overline{P E P}^{S C}=K L \sum_{l=0}^{L-1}\left(\begin{array}{c}
L-1 \\
l
\end{array}\right) \frac{(-1)^{l}}{l+1}-K L \sum_{l=0}^{L-1} \sum_{q=0}^{N-K} \\
\times\left(\begin{array}{c}
L-1 \\
l
\end{array}\right)\left(\begin{array}{c}
N-K \\
q
\end{array}\right) \frac{(-1)^{l+q}}{q(\bar{\gamma}+l+1)+l+1}, \\
\overline{P E P}_{\text {Approx. }}^{S C}=K L(N-K) \sum_{l=0}^{L-1}\left(\begin{array}{c}
L-1 \\
l
\end{array}\right) \frac{(-1)^{l}}{\bar{\gamma}+2 l+2} .
\end{gathered}
$$

A detailed description of the derivations are shown in the Appendix B.

As shown from (19) and (20), it can be obtained that the average PEP of the GD-SC relies on both $K$ and $L$, for given $N$ and $\bar{\gamma}$. Similar to the average PEP for MRC, it is revealed that (20) behaves concavely with $K$ for a given $N$ and $\bar{\gamma}$. This can be shown by taking the second derivative of $\overline{P E P}$ Approx. with respect to $K$, i.e., $\partial^{2}\left[\overline{P E P} \overline{A p p r o x}_{\text {Ap }}^{S C}\right] / \partial K^{2}<0 \forall K$. 


\section{Symbol Error Probability (SEP) Analysis}

\section{A. Instantaneous SEP for GD with Diversity Reception}

The SEP is defined as the probability of errors occurring in the detection of both the indices of active sub-carriers and M-ary symbols. These detection errors can occur from three error scenarios:

(i) A correct M-ary symbol conditioned on the incorrect detection of active sub-carrier index.

(ii) An incorrect $\mathrm{M}$-ary symbol conditioned on the incorrect detection of the active sub-carrier index.

(iii) An incorrect M-ary symbol conditioned on the correct detection of the active sub-carrier index.

Scenarios (i) and (ii) represent the conditional symbol errors (CSEs) that occur upon the mis-detection of the active indices, while Scenario (iii) represents the CSEs on the correct detection of active indices.

1) Probability of CSEs on Mis-Detection of Active Indices.: Scenario (i) can be defined as the probability of detecting an incorrect active index weighted by the probability of detecting a correct M-ary symbol, which is given as,

$$
Z_{e, i}=i P E P Z_{e_{s y m, i}},
$$

where $Z_{e_{s y m, i}}=1 / 2$ is the probability that a M-ary symbol is correctly determined without knowledge of $\mathrm{x}$ in (2), which represents a 50 percent detection accuracy of the M-ary symbol in the presence of the mis-detection of the active index, i.e., $\alpha \neq \tilde{\alpha} \forall \alpha, \tilde{\alpha}$.

Similarly, Scenario (ii) can be defined as the probability of detecting the incorrect active sub-carrier index weighted by the probability of detecting the incorrect M-ary symbol. The CSE probability in Scenario (ii) can be given as,

$$
Z_{e, i i}=i P E P Z_{e_{s y m, i i}},
$$

where $Z_{e_{s y m, i i}}=1 / 2$ is the probability that a M-ary symbol is incorrectly determined without knowledge of $\mathbf{x}$, i.e., $Z_{e_{s y m, i i}}=1 / 2$, which represents a 50 percent detection accuracy of the M-ary symbol in the presence of the misdetection of the active index, i.e., $\alpha \neq \tilde{\alpha} \forall \alpha, \tilde{\alpha}$. Therefore the CSEs on the mis-detection of active indices is given as,

$$
Z_{e, A}=Z_{e, i}+Z_{e, i i}=i P E P \text {. }
$$

2) Probability of CSEs on Correct Detection of Active Indices.: Intuitively Scenario (iii) can be defined as the probability of detecting a correct active index weighted by the probability of detecting an incorrect M-ary symbol;

$$
Z_{e, B}=\frac{K}{N} \sum_{\alpha}\left(1-i P E P\left(\gamma_{\beta, \alpha}\right)\right) \mathbf{P}\left(\gamma_{\beta, \alpha} \mid \mathbf{x}\right),
$$

where $\mathbf{P}\left(\gamma_{\beta, \alpha} \mid \mathbf{x}\right)$ is the SEP of the M-ary QAM [27]. For simplicity an approximation is used. Formally,

$$
\mathbf{P}\left(\gamma_{\beta, \alpha} \mid \mathbf{x}\right) \leq e^{\gamma_{\beta, \alpha}\left(-\frac{3}{2(M-1)}\right)} .
$$

Hence, using (23) and (25) the total SEP can be written as,

$$
\begin{aligned}
P_{s} & =Z_{e, A}+Z_{e, B}, \\
& =\frac{K}{N} \sum_{\alpha} i P E P\left(\gamma_{\beta, \alpha}\right)+\left(1-i P E P\left(\gamma_{\beta, \alpha}\right)\right) \mathrm{P}\left(\gamma_{\beta, \alpha} \mid \mathbf{x}\right) .
\end{aligned}
$$

Substituting (10) and (25) into (26), an expression for the instantaneous SEP is obtained as,

$$
\begin{aligned}
P_{s} & \leq \frac{K}{N} \sum_{\alpha=1}^{N}\left\{1-\sum_{q=0}^{N-K}\left(\begin{array}{c}
N-K \\
q
\end{array}\right) \frac{(-1)^{q}}{q+1} e^{-\frac{q \gamma_{\beta, \alpha}}{q+1}}+e^{-\frac{3 \gamma_{\beta, \alpha}}{2(M-1)}}\right. \\
& \left.-\sum_{q=0}^{N-K}\left(\begin{array}{c}
N-K \\
q
\end{array}\right) \frac{(-1)^{q}}{q+1} e^{-\frac{\gamma_{\beta, \alpha}(2 M q-q-3)}{2(M-1)(q+1)}}\right\}
\end{aligned}
$$

\section{B. Approximate Instantaneous SEP for GD with Diversity Reception}

An approximation of (27) can be obtained by substituting (11) and (25) into (26). Hence,

$$
\begin{aligned}
P_{s, \text { Approx. }} & =\frac{K}{N} \sum_{\alpha=1}^{N} \frac{N-K}{2}\left[e^{-\frac{\gamma_{\beta, \alpha}}{2}}-e^{-\frac{\gamma_{\beta, \alpha}(M+2)}{2(M-1)}}\right] \\
& +e^{-\frac{3 \gamma_{\beta, \alpha}}{2(M-1)}} .
\end{aligned}
$$

\section{Average SEP for GD-MRC}

Averaging (27) and (28) over (12) yields the expressions for the upper bounded and approximate average SEP (ASEP) for MCIK-OFDM GD-MRC, which can be formulated as,

$$
\bar{P}_{s}^{M R C}=\int_{0}^{\infty} P_{s}\left(\gamma_{\alpha}\right) f_{\gamma}\left(\gamma_{\alpha}\right) d \gamma_{\alpha} .
$$

The closed-form expressions for the upper bounded and approximate ASEP for GD-MRC are obtained, and given in (30) and (31), respectively, which can be found at the top of the next page. A detailed description of the derivations are shown in the Appendix C.

From (31) it is clear that the ASEP increases linearly with $N$ for a given $K, M, L$, and $\bar{\gamma}$. In addition, it is worth pointing out that the achievable diversity order is $d=$ $-\lim _{\bar{\gamma} \rightarrow \infty} \log \bar{P}_{s}^{M R C} / \log \bar{\gamma}=L$. Similar to (15), it can be found that (31) behaves concavely with $K$ for a given $N, M$, and $\bar{\gamma}$ as the second derivative of (31) is $\partial^{2}\left[\bar{P}_{s, A \text { pprox }}^{M R C}\right] / \partial K^{2}<0 \forall K$. Interestingly, it can also be obtained that (31) increases monotonically with $N$ for a given $K, M$, and $\bar{\gamma}$. Where monotonicity occurs when $\partial^{2}\left[\bar{P}_{s, A p p r o x}^{M R C}\right] / \partial N^{2}=0 \forall N$.

\section{GD-MRC: $L$ versus Maximum Tolerated ASEP $\mu$}

For reliable and low complexity systems, it is important to find the minimum number of $L$ so that the ASEP is guaranteed to be less than or equal to the maximum tolerated threshold $\mu$. Using $\bar{\gamma}=\frac{N}{K} \rho$, (31) can be re-written as,

$$
\begin{aligned}
\bar{P}_{s, \text { Approx. }}^{M R C} & =\left(\frac{2(M-1) K}{3 N \rho+2(M-1) K}\right)^{L} \\
& +K \frac{N-K}{2} \times\left[\left(\frac{2 K}{N \rho+2 K}\right)^{L}\right. \\
& \left.-\left(\frac{2(M-1) K}{N \rho(M+2)+2(M-1) K}\right)^{L}\right] .
\end{aligned}
$$

Denote by $L^{B C}$ the number of antennas that produces the ASEP less than or equal to $\mu$. $L^{B C}$ can be obtained as,

$$
L^{B C} \geq \arg \min _{L}\left|\bar{P}_{s}^{M R C}-\mu\right| .
$$




$$
\begin{aligned}
& \bar{P}_{s}^{M R C} \leq K\left[1-\sum_{q=0}^{N-K}\left(\begin{array}{c}
N-K \\
q
\end{array}\right) \frac{(-1)^{q}}{q+1}\left(\frac{q+1}{q(\bar{\gamma}+1)+1}\right)^{L}\right]+(1-K)\left(\frac{2(M-1)}{3 \bar{\gamma}-2(M-1)}\right)^{L} \\
&+K \sum_{q=0}^{N-K}\left(\begin{array}{c}
N-K \\
q
\end{array}\right) \frac{(-1)^{q}}{q+1}\left(\frac{2(M-1)(q+1)}{2 M q \bar{\gamma}+2 M q+2 M+\bar{\gamma}+3 \bar{\gamma}-2 q-2}\right)^{L} \\
& \bar{P}_{\text {s, Approx. }}^{\text {MRC }}=K \frac{N-K}{2}\left[\left(\frac{2}{\bar{\gamma}+2}\right)^{L}-\left(\frac{2(M-1)}{\bar{\gamma}(M+2)+2(M-1)}\right)^{L}\right]+\left(\frac{2(M-1)}{3 \bar{\gamma}+2(M-1)}\right)^{L}
\end{aligned}
$$

From (33) and (32), solving the equality of $\left|\bar{P}_{s}^{M R C}-\mu\right|=0$ for $L$, yields,

$$
\begin{aligned}
L^{*} & =\ln \{\mu\}\left[\ln \left\{K \frac{N-K}{2}\right\} \ln \left\{\frac{2 K}{N \rho+2 K}\right\}\right. \\
& -\ln \left\{K \frac{N-K}{2}\right\} \ln \left\{\frac{2(M-1) K}{N \rho(M+2)+2(M-1) K}\right\} \\
& \left.+\ln \left\{\frac{2(M-1) K}{3 N \rho+2(M-1) K}\right\}\right]^{-1} .
\end{aligned}
$$

Note that $L^{B C}$ in (33) must be an integer, and $L^{B C}$ may not be equal to $L^{*}$ from (34). Thus, using (34), the closedform expression for the minimum desired number of antennas $L$ satisfying the ASEP less than $\mu$, is shown in (35). Since $\mu(\leq 1)$ is the desired error probability, it can be found from (35) that as $\mu$ increases, $L^{B C}$ decreases logarithmically for given $N, K, M$, and $\rho$.

\section{E. Average SEP for GD-SC}

Averaging (27) and (28) over (17) yields the expressions for the upper bounded and approximate average SEP for MCIKOFDM GD-SC, which can be formulated as,

$$
\bar{P}_{s}^{M R C}=\int_{0}^{\infty} P_{s}\left(\gamma_{\alpha}\right) f_{\gamma}\left(\gamma_{\alpha}\right) d \gamma_{\alpha} .
$$

Closed-form expressions for the upper bounded and approximate ASEP for GD-SC are obtained, which are given in (37) and (38), respectively. A detailed description of the derivations are shown in the Appendix D. Similar to (19) and (20) the complexity of (37) and (38) is high due to the multiple summations and binomial coefficient terms, and as a result detailed analysis is not straight forward. It can be found that (38) increases monotonically with $N$ for a given $K, M$, and $\bar{\gamma}$. Where monotonicity occurs when $\partial^{2}\left[\bar{P}_{s, \text { Approx }}^{S C}\right] / \partial N^{2}=0 \forall N$.

\section{Asymptotic AnALYsis}

In this section various asymptotic cases are considered in order to investigate the asymptotic behaviour of MCIK-OFDM with GD-MRC in terms of the ASEP. For simplicity, only the approximate expression (31) is considered, which still holds its accuracy for the considered asymptotic cases.

\section{A. Large $L: L \rightarrow \infty$}

Consider a case where there is a large antenna array at the receiver. Let $K=\tau N$ be an integer with a fixed $\tau$. This means that when $N$ is increased $K$ also does, but the ratio of $K$ to $N$ is fixed to $\tau$. In terms of $\tau$, the approximate ASEP for MRC is given as,

$$
\begin{aligned}
& \bar{P}_{s, \text { Approx. }}^{M R C}=\left(\frac{2(M-1) \tau}{3 \rho+2(M-1) \tau}\right)^{L}+N^{2} \frac{\tau-\tau^{2}}{2} \\
& \times\left[\left(\frac{2 \tau}{\rho+2 \tau}\right)^{L}-\left(\frac{2(M-1) \tau}{\rho(M+2)+2(M-1) \tau}\right)^{L}\right] .
\end{aligned}
$$

In this case, i.e., $L \rightarrow \infty$, the ASEP for high $\rho$ is given as,

$$
\begin{aligned}
\lim _{L \rightarrow \infty} \bar{P}_{\text {s,Approx. }}^{\text {MRC }} & =c\left[\left(\frac{2 \tau}{\rho}\right)^{L}-\left(\frac{2 \tau(M-1)}{\rho(M+2)}\right)^{L}\right] \\
& +\left(\frac{2 \tau(M-1)}{3 \rho}\right)^{L}, \\
& =\Theta\left((M \tau)^{L} S N R^{-L}\right),
\end{aligned}
$$

where $S N R=\rho$, and $c$ is a constant.

From (40) it can be asymptotically observed that for large $L$, the ASEP decreases exponentially with decreasing $\tau=K / \mathrm{N}$ for given $M$ and $\rho$. Intuitively, this suggests that choosing a small $\tau$ will reduce the ASEP exponentially at large $L \mathrm{~s}$, for given $N, M$, and $\rho$. In addition, it is shown from (40) that the ASEP increases exponentially with $M$ for given $\tau$ and $\rho$. Therefore, the ASEP in (40) benefits from properly having $\tau=K / N(\leq 1)$ for large $L$, which leads to the achievable power gain greater than the classical OFDM $(\tau=1)$.

\section{B. Small Sparsity Ratio: $\tau \rightarrow 0$}

To address the impact of $\tau$ on the ASEP. For example, in D2D communications low complexity is a key design parameter. In this context, reducing $K$ in turn reduces the decoding complexity. Therefore, as $N$ grows, consider a very small $\tau$, i.e., when $K \ll N$. In this asymptotic case the first two terms of (31) tend to zero much faster than the third term, i.e., $N^{2} \frac{\tau-\tau^{2}}{2}\left[\left(\frac{2 \tau}{\rho}\right)^{L}-\left(\frac{2 \tau(M-1)}{\rho(M+2)}\right)^{L}\right] \rightarrow 0$, hence the ASEP for this asymptotic case can be reduced as,

$$
\begin{aligned}
\lim _{\tau \rightarrow 0} \bar{P}_{s, \text { Approx. }}^{\text {MRC }} & =\left(\frac{2(M-1) \tau}{3 \rho}\right)^{L} \\
& =\left(\frac{\tau}{\rho}\right)^{L}(M-1)^{L} c \\
& =\Theta\left(\tau^{L} S N R^{-L}\right)
\end{aligned}
$$




$$
L^{B C}=\left\lceil\frac{\ln \{\mu\}}{\ln \left\{K \frac{N-K}{2}\right\}\left(\ln \left\{\frac{2 K}{N \rho+2 K}\right\}-\ln \left\{\frac{2(M-1) K}{N \rho(M+2)+2(M-1) K}\right\}\right)+\ln \left\{\frac{2(M-1) K}{3 N \rho+2(M-1) K}\right\}}\right\rceil
$$

$$
\begin{aligned}
& \bar{P}_{s}^{S C} \leq K \sum_{l=0}^{L-1}\left(\begin{array}{c}
L-1 \\
l
\end{array}\right)\left\{(-1)^{l}\left[\frac{L}{l+1}+\frac{2(M-1) L}{3 \bar{\gamma}+2(M-1)(l+1)}\right]-\sum_{q=0}^{N-K}\left(\begin{array}{c}
N-K \\
q
\end{array}\right)(-1)^{q+l}\right. \\
& {\left.\left[\frac{L}{q(\bar{\gamma}+l+1)+l+1}+\frac{2(M-1) L}{\bar{\gamma}(2 M q+q+3)+2 l M q+2 l M-2 l q+2 M q+2 M-2 l-2 q-2}\right]\right\} } \\
& \bar{P}_{s, \text { Approx. }}^{S C}=K(N-K) L \sum_{l=0}^{L-1}\left(\begin{array}{c}
L-1 \\
l
\end{array}\right) \frac{(-1)^{l}}{\bar{\gamma}-2 l+2}+L \sum_{l=0}^{L-1}\left(\begin{array}{c}
L-1 \\
l
\end{array}\right)(-1)^{l} \\
& \quad \times \frac{2(M-1)}{3 \bar{\gamma}+2(M-1)(l+1)}-K(N-K) L \sum_{l=0}^{L-1}\left(\begin{array}{c}
L-1 \\
l
\end{array}\right)(-1)^{l} \frac{M-1}{\bar{\gamma}(M+2)+2(M-1)(l+1)}
\end{aligned}
$$

Asymptotically it is observed from (41) that the ASEP achieves the diversity order $L$ for given $\tau$ and $\rho$. Where $\tau \in(0,1]$, a small $\tau$ will reduce the ASEP faster than a large $\tau$ for a given $L$. As a result, a proper choice of joint small $\tau$ and large $L$ can make the ASEP decrease for a given $\rho$. Therefore even in low and moderate SNR regions a reasonable ASEP can be achieved by choosing the smallest $\tau$.

\section{Large Sparsity Ratio: $\tau \rightarrow 1$}

As $K$ approaches $N, \tau$ also increases, i.e., $\tau \rightarrow 1$. In this case the ASEP can be expressed as

$$
\begin{aligned}
\lim _{\tau \rightarrow 1} \bar{P}_{s, \text { Approx }}^{M R C} & =\left(\frac{2(M-1) \tau}{3 \rho+2(M-1) \tau}\right)^{L}, \\
& =\left[\Theta\left(\frac{S N R}{(M-1) \tau}\right)+1\right]^{-L} .
\end{aligned}
$$

In this case when $K \rightarrow N$, MCIK-OFDM begins to behave like OFDM. This leads that choosing the smallest $M$ will reduce the ASEP, for a given $L$ and $\rho$.

\section{Computational Complexity}

We now address the decoding complexity of the hybrid GD and diversity reception for MCIK-OFDM in terms of the total number of complex multiplications and divisions (CMs) performed per cluster. This will be compared to the ML method. The CMs for various detectors are summarized in table II.

First, let us consider the decoding complexity of ML in [28]. The ML needs a brute-force search for all active sub-carrier index combinations and corresponding M-ary symbols. The decoding complexity of ML is given as,

$$
\mathcal{C}_{M L}=2 C M^{K},
$$

where $C=2^{\left\lfloor\log _{2}\left(\begin{array}{l}N \\ K\end{array}\right)\right\rfloor}$ is recalled as the total number of active sub-carrier index combinations, and $M^{K}$ is the number of Mary symbol combinations across $K$ active sub-carriers, for each active index combination. In total there are $C M^{K}$ trials needed in the ML method and $2 \mathrm{CMs}$ per trial. Note that evaluating the Euclidean distance in (7) requires 8 real multiplications and hence a total of $2 \mathrm{CMs}$ per trial. As a result, the classical ML detection becomes impractical for larger values of $K$ due to its exponentially growing decoding complexity with $K$.

Unlike the ML, the proposed GD scheme performs the twostage process which first finds the active sub-carrier index followed by the M-ary symbol. The decoding complexity of GD is,

$$
\mathcal{C}_{G D}=N+2 K M
$$

where $N \mathrm{CMs}$ are required to perform the energy detection of active sub-carriers, requiring $1 \mathrm{CM}$ to evaluate the energy per sub-carrier $\left(|y|^{2}\right)$ in (6), and $2 K M \mathrm{CMs}$ are required to perform ML detection of data symbols, given there are $2 \mathrm{CMs}$ per trial, with $M$ trials, for $K$ active sub-carriers. It is worth pointing out from (44) that the complexity of GD increases linearly with $K$, while that of ML in (43) grows exponentially with $K$. Hence, the GD is a low complexity alternative to the ML.

Similar to the GD, the log-likelihood ratio (LLR) detector, first introduced in [9], performs a two-stage detection process. First, the LLR test determines the active subcarrier indices, followed by ML detection of the data symbols. Hence, the decoding complexity of LLR is,

$$
C_{L L R}=2 N+2 N M+2 K M,
$$

where there are $2+2 M$ CMs required for the LLR test per sub-carrier, for a total of $N$ sub-carriers. From [9, (12)] there are 2 CMs required for $\left(|y|^{2} / N_{0}\right)$ and an addition $2 M$ required for $\left(\sum_{m=1}^{M}\left|y-h x_{m}\right|^{2} / N_{0}\right)$. In addition, there are $2 K M \mathrm{CMs}$ required to perform ML detection of data symbols. The detection complexity of the active sub-carrier indices alone, can be given as $\sim \mathcal{O}(N M)$, whereas the GD is $\sim \mathcal{O}(N)$. Hence, the complexity of GD is reduced by a factor of $M$ over the LLR.

The decoding complexity of the well known ML for a 
TABLE II

Number of COMPlex Multiplications Per Cluster

\begin{tabular}{|c|c|c|}
\hline Detection Scheme & Decoding Complexity & Example: $(N, K, M, L)=(4,3,4,2)$ \\
\hline \hline MCIK-OFDM ML & $2 C M^{K}$ & 512 \\
\hline GD & $N+2 K M$ & 28 \\
\hline LLR & $2 N+2 N M+2 K M$ & 64 \\
\hline OFDM ML & $2 N M$ & 32 \\
\hline MCIK-OFDM ML-SC & $N+2 C M^{K}$ & 516 \\
\hline MCIK-OFDM ML-MRC & $L N+2 C M^{K}$ & 520 \\
\hline OFDM ML-SC & $N+2 N M$ & 36 \\
\hline OFDM ML-MRC & $L N+2 N M$ & 40 \\
\hline GD-SC & $N+N+2 K M$ & 32 \\
\hline GD-MRC & $L N+N+2 K M$ & 36 \\
\hline LLR-SC & $N+2 N+2 N M+2 K M$ & 68 \\
\hline LLR-MRC & $L N+2 N+2 N M+2 K M$ & 72 \\
\hline
\end{tabular}

conventional OFDM system is defined as,

$$
\mathcal{C}_{O F D M-M L}=2 N M,
$$

where $2 N M \mathrm{CMs}$ are required to recover data symbols using the ML decision, given there are $2 \mathrm{CMs}$ per trial, with $M$ trials, for $N$ sub-carriers.

Regarding to the use of diversity combining, the complexity of both ML and GD are increased by that of the diversity combining. Particularly, the CMs required for ML-SC and ML-MRC are given respectively as,

$$
\begin{gathered}
\mathcal{C}_{M L-S C}=N+2 C M^{K}, \\
\mathcal{C}_{M L-M R C}=L N+2 C M^{K},
\end{gathered}
$$

where the additional $N$ and $L N$ CMs resulting from the diversity combining are included for ML-MRC and ML-SC, respectively.

Similarly, the CMs needed for ML-SC and ML-MRC for a conventional OFDM are respectively given as,

$$
\begin{gathered}
\mathcal{C}_{O F D M-M L-S C}=N+2 N M, \\
\mathcal{C}_{O F D M-M L-M R C}=L N+2 N M,
\end{gathered}
$$

and the decoding complexity of GD-SC, GD-MRC, LLR-SC, and LLR-MRC for MCIK-OFDM are defined as, respectively,

$$
\begin{gathered}
\mathcal{C}_{G D-S C}=N+N+2 K M, \\
\mathcal{C}_{G D-M R C}=L N+N+2 K M, \\
\mathcal{C}_{L L R-S C}=N+2 N+2 N M+2 K M, \\
\mathcal{C}_{L L R-M R C}=L N+2 N+2 N M+2 K M .
\end{gathered}
$$

For example, consider an MCIK-OFDM system with $N=$ $8, K=4$, and $M=4$. The number of $\mathrm{CMs}$ required for ML is 32768, while the number of CMs for GD is 44. The number of CMs performed for GD is significantly reduced by a factor of over 700, over the ML. Moreover, considering the hybrid GD and diversity reception with $L$ diversity branches, the number of additional CMs by MRC or SC are affordable, increasing linearly with $N$. Therefore, GD with diversity reception is promising for low-complexity MCIK-OFDM applications while ML becomes impractical due to its exponentially increasing complexity.
For comparison between the MCIK-OFDM and the conventional OFDM, we can observe in Table II that satisfying $\widetilde{K} \leq N(M-1 / 2) / M$ in the MCIK-OFDM always produces the decoding complexity less than or equal to that of OFDM, and the corresponding data-rate is computed, as $\left\lfloor\log _{2}\left(\begin{array}{c}N \\ \widetilde{K}\end{array}\right)\right\rfloor+\widetilde{K} \log _{2} M$, where $\widetilde{K}$ is the largest integer not greater than $N(M-1 / 2) / M$.

\section{NUMERICAL RESULTS AND DISCUSSION}

In this section, numerical and simulation results for the MCIK-OFDM scheme with various detection and diversity reception methods are presented. The MCIK-OFDM system with $N_{c}=128$ total sub-carriers, grouped into $G$ clusters of $N$ sub-carriers, where $N \in\{4,8\}, K \leq 8, L \in\{1,2,4,8\}$, and $M \in\{2,4,8,16\}$. As each cluster of $N$ sub-carriers is detected individually, $N_{c}$ has no effect on the performance, and the number of $N$ is chosen to be much less than $N_{c}$ for a low-complexity detection process.

Fig. 3 depicts the average SEP performance of GD-MRC, for MCIK-OFDM with $N=4, K=1, M=4$, and $L=\{1,2,4,8\}$. It can be observed that the SEP performance is significantly improved as $L$ is increased. For example, the power gain of about $8 \mathrm{~dB}$ can be achieved for the average SEP of $10^{-3}$ between $L=2$ and $L=4$. Moreover, it can be found from this figure that the analytical results display their high accuracy in a range of SNRs, e.g, $2 \mathrm{~dB}$ difference from simulations for the average SEP of $10^{-2}$ and $L=1$.

In Fig. 4 a comparison between the upper bounded and approximate average SEP expressions for GD-MRC is illustrated for the MCIK-OFDM system when $N=4, K=1$, $M=4$, and $L=\{1,2,4,8\}$. The maximum difference between (30) and (31) is observed to remain less than $1 \mathrm{~dB}$ in this figure. This confirms the tightness of the approximate expression. Moreover, the approximate expression becomes tighter as $L$ increases. This validates the accuracy of the approximate SEP.

Fig. 5 depicts the behaviour of the average SEP against $\tau=K / N$, for MCIK-OFDM GD-MRC with $N=8, M=$ $\{4,8,16,32\}, L=2$, and $E_{s} / N_{0}=10 \mathrm{~dB}$. It can be observed from the figure that the average SEP behaves concavely with $K$ for a given $N$ and monotonically increases with $\tau$ for a larger $M$ (i.e., $M=16$ ). This observation validates the 


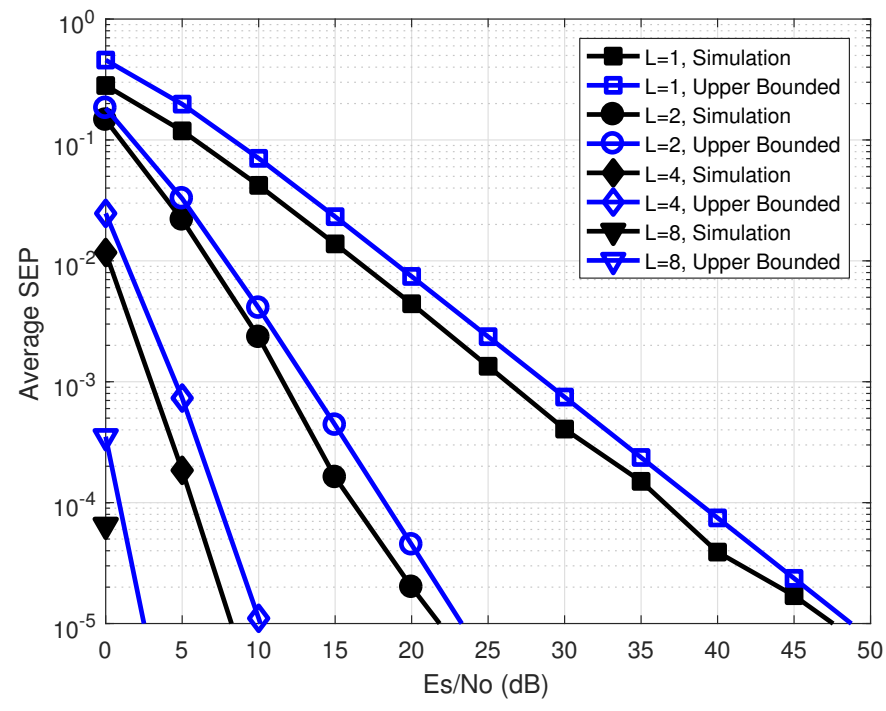

Fig. 3. Average SEP performance of MCIK-OFDM with GD-MRC for $N=$ $4, K=1, M=4$, and $L=\{1,2,4,8\}$.

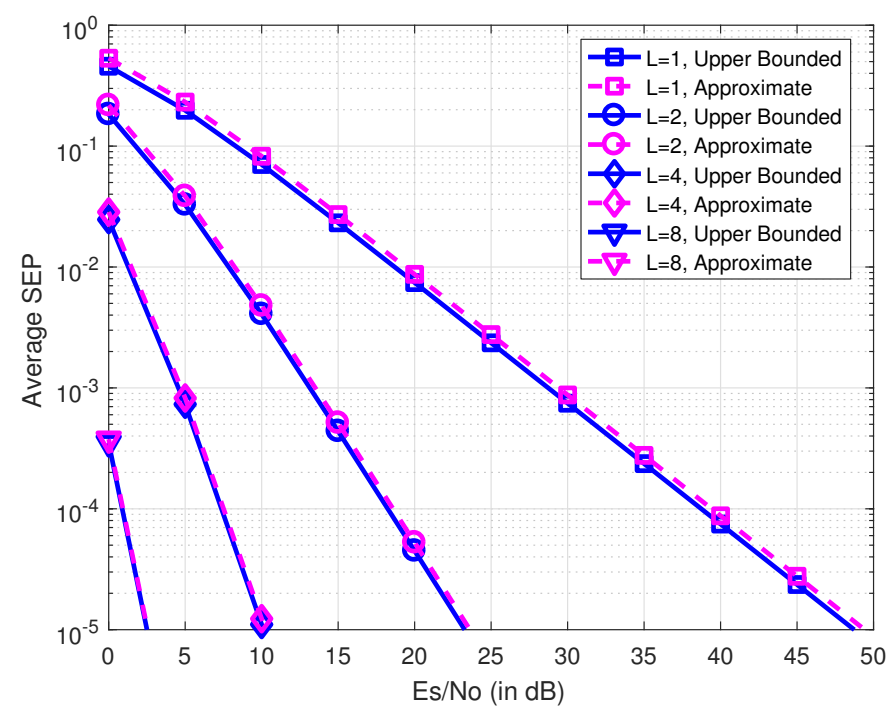

Fig. 4. Comparison of upper bounded and approximate average SEP for MCIK-OFDM with GD-MRC for $N=4, K=1, M=4$, and $L=$ $\{1,2,4,8\}$.

analysis in (31). Also, it can be found from this figure that the upper bounded results display their tightness to the simulated results, e.g., for $\tau=0.9$ there is an average SEP difference of greater than $4 \times 10^{-2}$. It is worth pointing out that the tightness of the expression increases as $M$ grows.

Fig. 6 illustrates now the average PEP performance of GD-MRC, for MCIK-OFDM with $N=4, K=1$, and $L=\{1,2,4,8\}$. Similar to the average SEP, it is shown that the average PEP is substantially improved as $L$ increases. For example, the power gain of up to $15 \mathrm{~dB}$ can be achieved for the average PEP of $10^{-3}$ between $L=1$ and $L=2$. In this figure the maximum gap of $1 \mathrm{~dB}$ between the simulations and (14) is observed which confirms the tightness of the exact expression. Furthermore, the maximum gap of less than $2 \mathrm{~dB}$ between (14) and (15) is observed, which validates

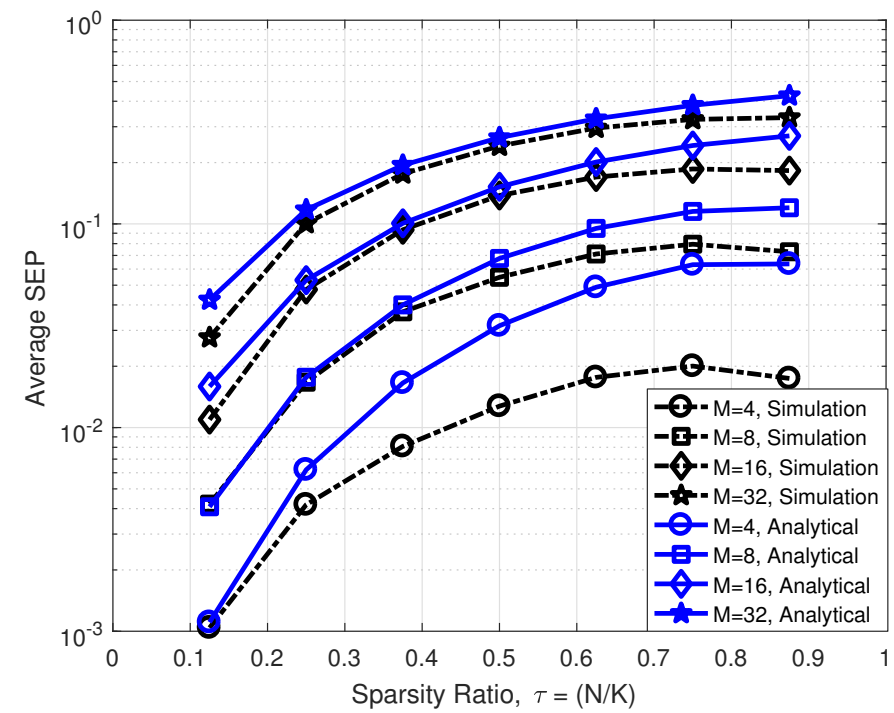

Fig. 5. Impact of $\tau$ on the average SEP for MCIK-OFDM with GD-MRC for $N=8, M=\{4,8,16,32\}, L=2$, and $E_{s} / N_{0}=10 \mathrm{~dB}$.

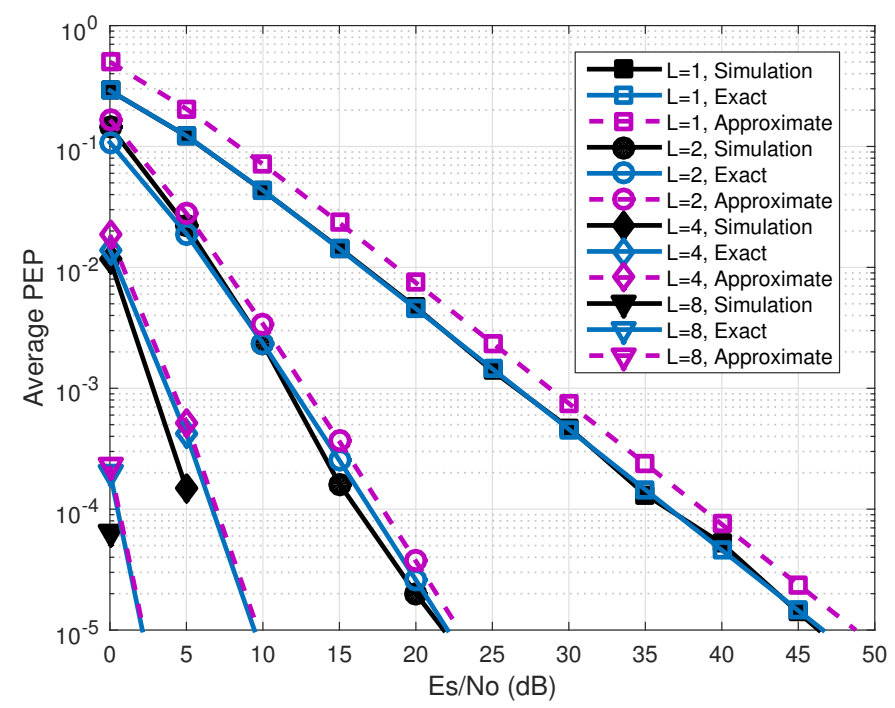

Fig. 6. Average PEP performance of MCIK-OFDM with GD-MRC for $N=$ $4, K=1$, and $L=\{1,2,4,8\}$.

the tightness of the approximate expression. Moreover, in Fig. 6 the approximate expression becomes tighter for large $L(\geq 2)$, providing less than $1 \mathrm{~dB}$ difference between exact and approximate expressions.

In Fig. 7, considering GD-SC, the average SEP of MCIKOFDM has been depicted when $N=4, K=1, M=4$, and $L=\{1,2,4,8\}$. It can be observed that as the number of diversity branches is increased, the average SEP improves significantly with the diversity order $L$. For example, the power gain of up to $6 \mathrm{~dB}$ can be achieved for the average SEP of $10^{-3}$ between $L=2$ and $L=4$, while the largest power gain of $13 \mathrm{~dB}$ is achieved between $L=1$ and $L=2$, for the average SEP of $10^{-3}$. Unlike the GD-MRC, Fig. 7 shows the power gain of GD-SC increasing slowly as $L$ is increased. Furthermore, the maximum difference of $2 \mathrm{~dB}$ between the simulations and (37) is observed, which validates the tightness 


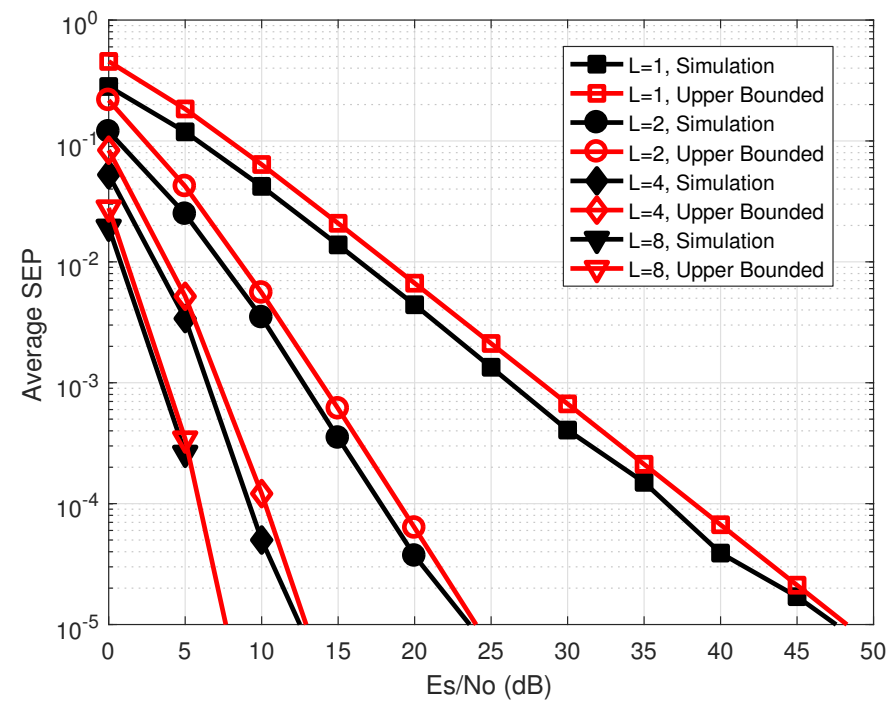

Fig. 7. Impact of $\tau$ on the average SEP for MCIK-OFDM with GD-SC for $N=4, K=1, M=4$, and $L=\{1,2,4,8\}$.

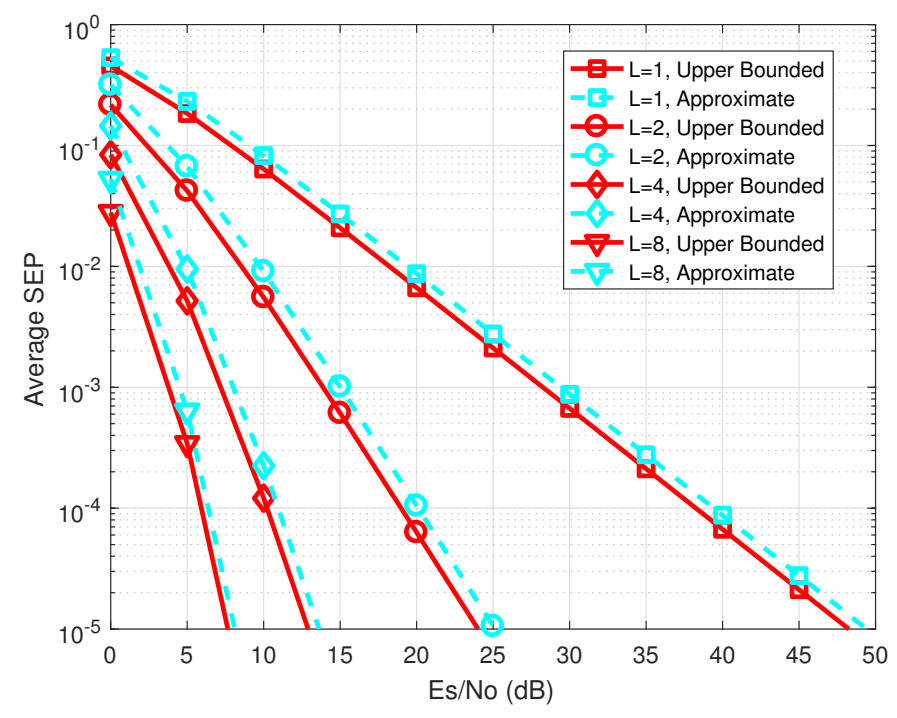

Fig. 8. Comparison of upper bounded and approximate average SEP for MCIK-OFDM with GD-SC for $N=4, K=1, M=4$, and $L=$ $\{1,2,4,8\}$.

of the upper bounded expression. This tightness of the upper bounded results improves as $L$ increases.

As for GD-SC, Fig. 8 compares the upper bounded and approximate average SEP of an MCIK-OFDM system with $N=4, K=1, M=4$, and $L=\{1,2,4,8\}$. The maximum difference of $1 \mathrm{~dB}$ between (37) and (38) is observed in a range of SNRs. This confirms the tightness of the approximate expression. Moreover, the approximate expression becomes tighter $(\leq 1 \mathrm{~dB})$ as $L$ increases.

In Fig. 9 the concave behaviour of the average SEP against $\tau=K / N$ is shown, for MCIK-OFDM GD-SC with $N=8$, $L=2, E_{s} / N_{0}=10 \mathrm{~dB}$, and $M=\{4,8,16,32\}$. This observation validates the analysis on the concavity of (38) with $K$. Due to this concave property it is worthy to mention from this figure that there may exist various choices of $(M, \tau)$ 's

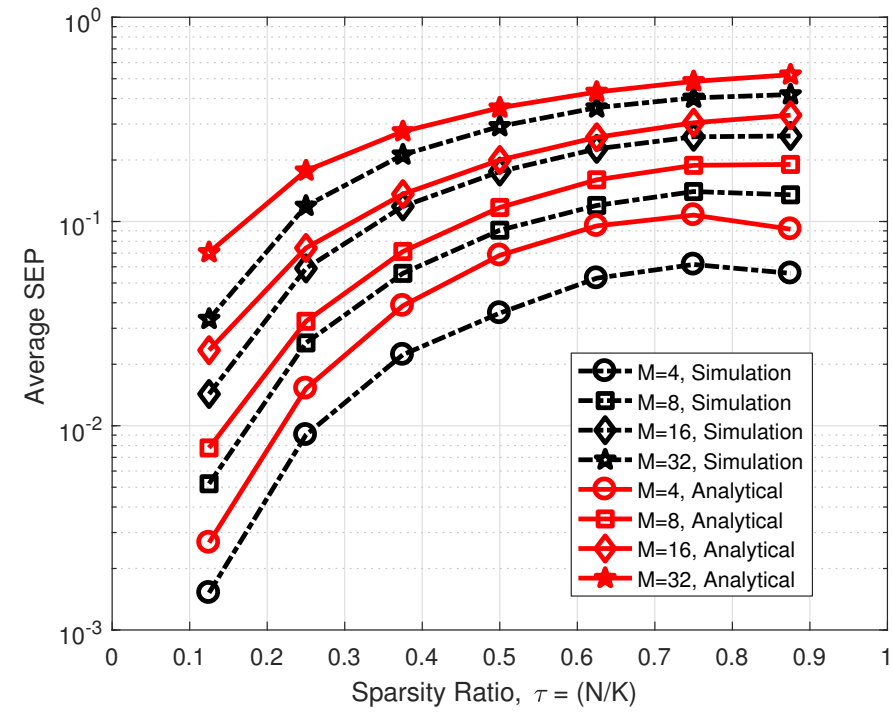

Fig. 9. Average SEP versus $\tau$ for MCIK-OFDM with GD-SC for $N=8$, $M=\{4,8,16,32\}, L=2$, and $E_{s} / N_{0}=10 \mathrm{~dB}$

satisfying a certain requirement of the average SEP, e.g., average SEP $\leq 10^{-2}$. Also, in this figure the upper bounded results display their tightness to the simulation results, e.g., for $\tau=0.5$ there is an average SEP difference of less than $2 \times 10^{-2}$. It is worth noting that the tightness of the expression increases as $M$ is increased.

Fig. 10 illustrates the performance of GD-SC, for MCIKOFDM with $N=4, K=1$, and $L=\{1,2,4,8\}$. Similar the average SEP of GD-SC, it can be observed from this figure that the average PEP improves as $L$ increases. For example, it is shown that the achievable diversity order equals 1 . Also, the power gain of up to $13 \mathrm{~dB}$ can be achieved for the average PEP of $10^{-3}$ between $L=1$ and $L=2$. The maximum difference of less than $1 \mathrm{~dB}$ between the simulations and (19) is observed which confirms the tightness of the exact expression. Furthermore, this figure depicts the maximum difference of less than $2 \mathrm{~dB}$ between (19) and (20) in a range of SNRs which confirms the tightness of the approximate expression. The approximate expression becomes tighter as $L$ increases, achieving a less than $1 \mathrm{~dB}$ difference between exact and approximate expressions.

Fig. 11 now illustrates the comparison between MCIKOFDM with GD-MRC and conventional OFDM with MLMRC in terms of average SEP, for $E_{s} / N_{0}=\{5,10,15\} \mathrm{dB}$, and $L=\{1, \ldots, 8\}$. The OFDM with ML-MRC is chosen to provide the best SEP of the classical OFDM. For a fair comparison the transmission data-rate of both schemes are set to be same, i.e., 8 bits per cluster. Interestingly, Fig. 11 shows that MCIK-OFDM with GD-MRC can outperform OFDM with ML-MRC in terms of the average SEP for a certain range of $L$, which varies with the SNR. In particular, when $E_{s} / N_{0}=5 \mathrm{~dB}$, the proposed scheme outperforms the OFDM when $L>4$. In addition, for high SNRs it shows that the number of $L$ needed for MCIK-OFDM to outperform OFDM decreases, which validates our analysis in (35). In particular, for $E_{s} / N_{0}=15 \mathrm{~dB}$, Fig. 11 shows that we have $L^{B C}=2$, 


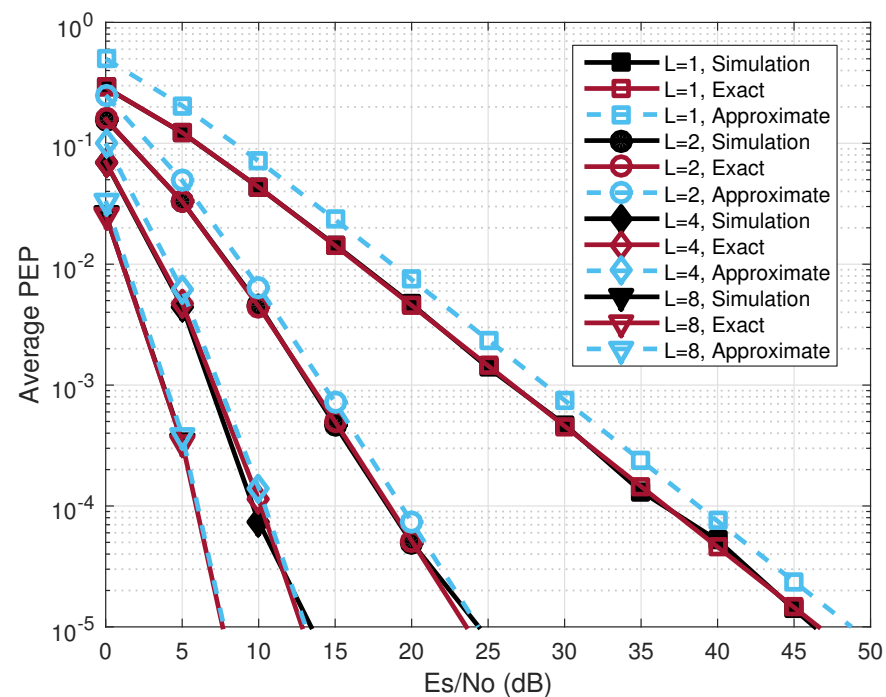

Fig. 10. Average PEP performance of MCIK-OFDM with GD-SC for $N=4$, $K=1$, and $L=\{1,2,4,8\}$.

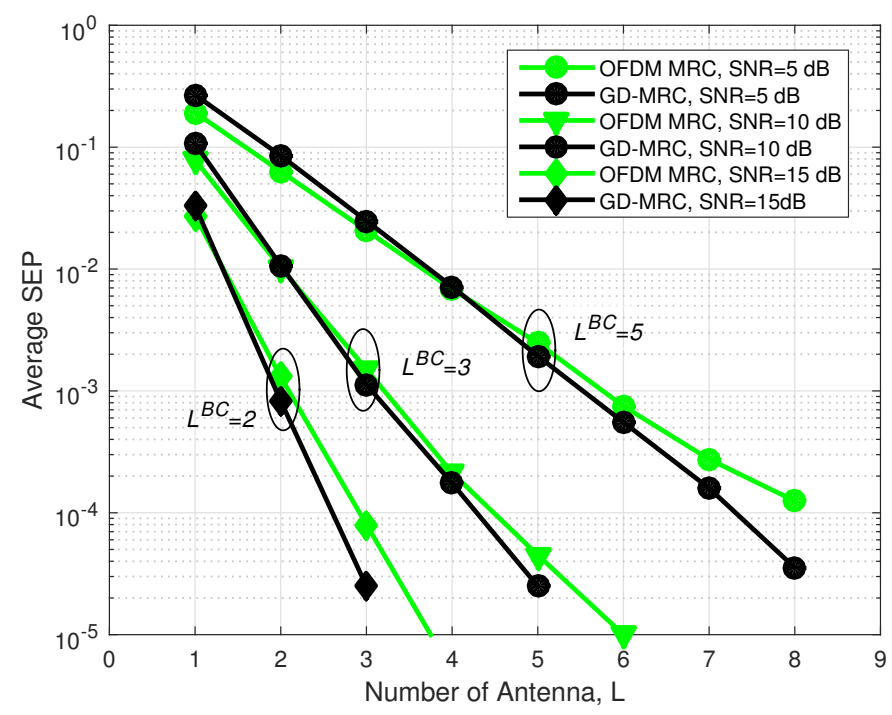

Fig. 11. Comparison between MCIK-OFDM with GD-MRC and conventional OFDM with ML-MRC at the same data-rate. $N=4, K=3, M=4$, $L=\{1, \ldots, 8\}$, and $E_{s} / N_{0}=\{5,10,15\} \mathrm{dB}$.

i.e., MCIK-OFDM with GD-MRC outperforms OFDM with ML-MRC when $L \geq 2$, even at the same data-rate.

Similarly, Fig. 12 depicts the average SEP comparison between MCIK-OFDM with GD-SC and conventional OFDM with ML-SC, for $E_{s} / N_{0}=\{5,10,15\} \mathrm{dB}$, and $L=\{1, \ldots, 8\}$. As in the previous figure, the transmission data-rate of both schemes remains the same, i.e., 8 bits per cluster. Fig. 12 depicts that MCIK-OFDM with GD-SC can outperform OFDM with ML-SC for a certain range of $L$. As the SNR is increased the number of $L$ needed for MCIK-OFDM to outperform OFDM is shown to decrease.

In Fig. 13, the simulated average SEP performance of GD, GD-MRC, and GD-SC are compared with LLR, LLR-MRC, and LLR-SC for an MCIK-OFDM system with $N=4$, $K=2, M=4$, and $L=\{1,2\}$. It can be observed in

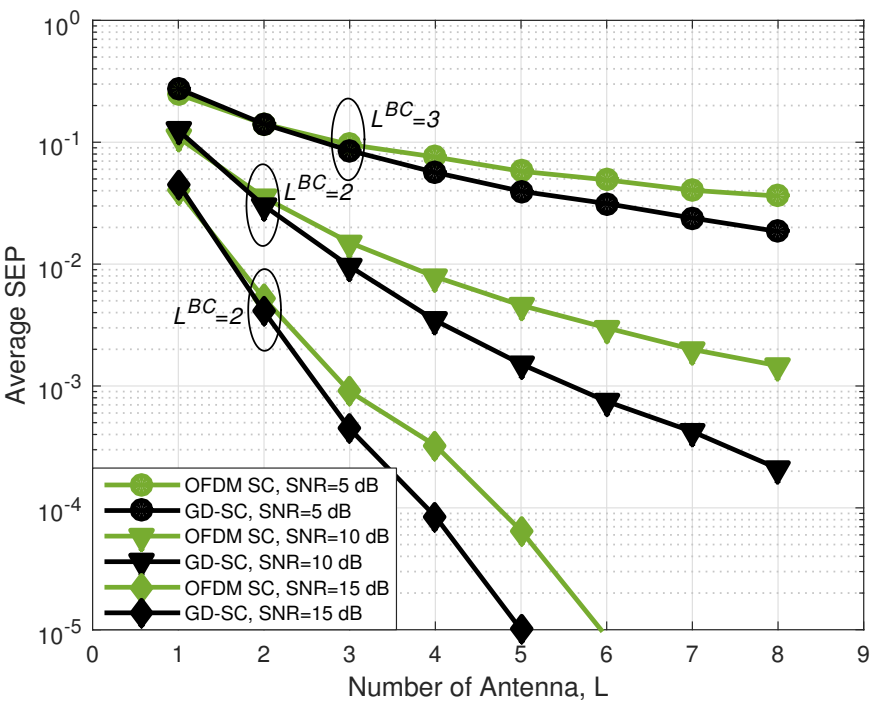

Fig. 12. Comparison between MCIK-OFDM with GD-SC and conventional OFDM with ML-SC at the same data-rate. $N=4, K=3, M=4$, $L=\{1, \ldots, 8\}$, and $E_{s} / N_{0}=\{5,10,15\} \mathrm{dB}$.

Fig. 13 that the LLR outperforms the GD. Specifically, for the average SEP of $10^{-3}$ there is the power gain of $5 \mathrm{~dB}$ for LLR over GD. However, such power gain attained by LLR-MRC (or LLR-SC) over GD methods is reduced when $L$ grows. In particular, for the average SEP of $10^{-3}$, and when $L=2$, there is a power gain of $3.5 \mathrm{~dB}$ for LLR$\mathrm{SC}$ over GD-SC, and a power gain $0.5 \mathrm{~dB}$ for LLR-MRC over GD-MRC. Referring to the expressions in Table II, there are 28, 24, and $20 \mathrm{CMs}$ involved in the GD-MRC, GD-SC, and GD detection processes, respectively. While there are 64, 60, and 56 CMs involved in LLR-MRC, LLR-SC, and LLR detection processes, respectively. This observation implies that GD-MRC (or GD-SC) still provides an affordable complexity less than the LLR $(L=1)$, even at better average SEP. Hence, even at small values of $N, K$, and $M$, it is shown that the proposed schemes can outperform the LLR in terms of both complexity and average SEP performance.

Fig. 14 compares the average SEP performance of GDMRC (and GD-SC) and ML-MRC (and ML-SC) for an MCIK-OFDM system with $N=4, K=2, M=4$, and $L=\{2,4,8\}$. It can be observed from the figure that MLMRC only marginally outperforms GD-MRC. Specifically, for the average SEP of $10^{-3}$, and $L=2$, ML-MRC achieves a power gain of less than $1 \mathrm{~dB}$ over GD-MRC. Moreover, as $L>2$ grows the performance gap between GD-MRC and ML-MRC becomes smaller. In particular, when $L=8$ the difference between GD-MRC and ML-MRC is negligible. In terms of decoding complexity, there are 136 and $28 \mathrm{CMs}$ involved in the ML-MRC and GD-MRC detection processes, respectively. Interestingly, this proves that for $L>1$, GDMRC is preferable over ML-MRC as it performs close to the optimal ML-MRC while maintaining a significantly reduced complexity. Similarly, we can observe from this figure that the GD-SC at the low complexity performs closer to the ML-SC, as $L$ grows. 


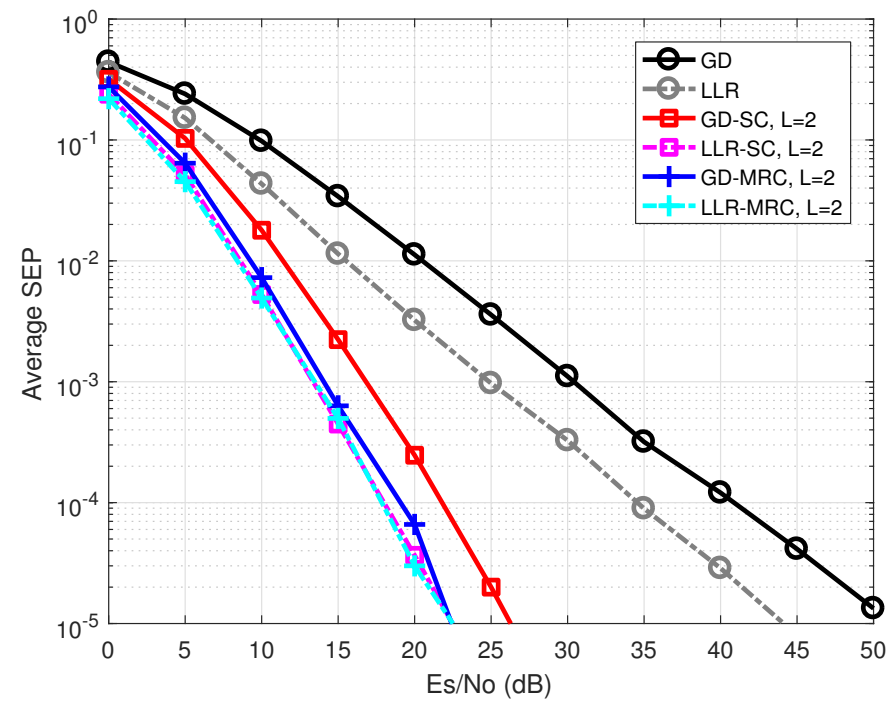

Fig. 13. Comparison of average SEP of MCIK-OFDM system with various detectors (GD, GD-MRC, GD-SC, LLR, LLR-MRC, LLR-SC) where $N=4$, $K=2, M=4$, and $L=\{1,2\}$.

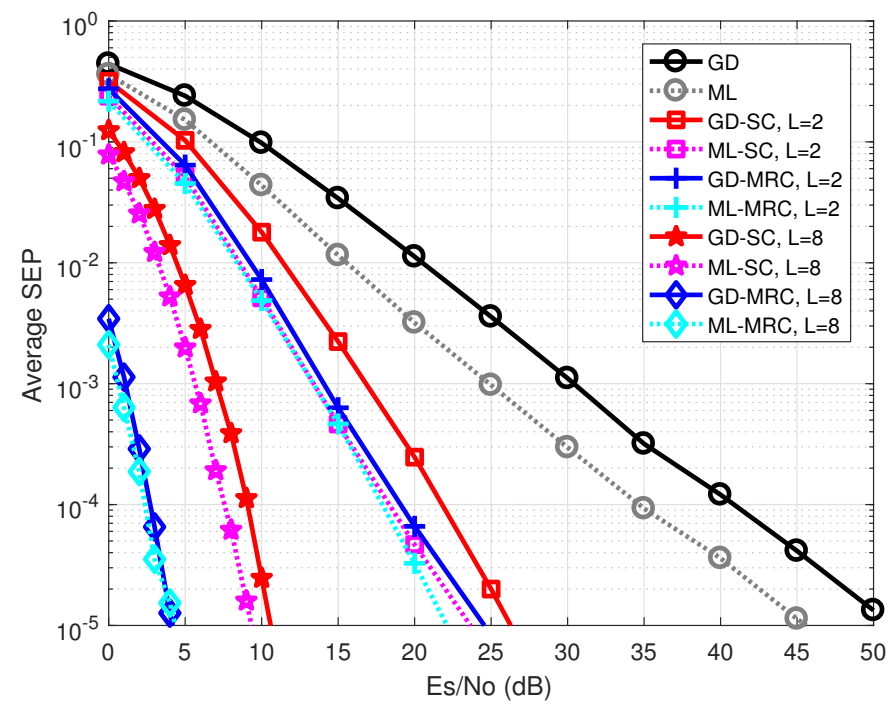

Fig. 14. Comparison of average SEP of MCIK-OFDM system with various detectors (GD, GD-MRC, GD-SC, ML, ML-MRC, ML-SC) where $N=4$, $K=2, M=4$, and $L=\{2,4,8\}$.

\section{CONCLUSIONS}

We proposed the new low complexity detection scheme for the MCIK-OFDM that exploits jointly the energy detection of active sub-carrier indices and two different diversity receptions. For the performance evaluation, closed-form expressions for the average PEP and the average SEP of the MCIKOFDM were derived, taking into account the three detection approaches: GD, GD-MRC, and GD-SC. Furthermore, the approximate and accurate SEP expressions have been derived in closed form. The derived expressions provided the insight into the impact of sub-carrier index activation and multiple antennas on the average SEP in low, moderate and extreme rate of the sparse sub-carrier index activation. The theoretical analysis and numerous comparisons have clearly shown that with the proposed hybrid GD and diversity reception, the MCIK-OFDM outperforms the conventional OFDM in terms of the reliability at significantly reduced complexity. The derived average SEP expressions will be useful to evaluate various concepts of the MCIK-OFDM, especially for future device applications that demands high reliability at low complexity. Our future work will be directed towards considering more fading conditions appropriate for D2D communications systems.

\section{APPENDIX A \\ DERIVATION OF (14) AND (15)}

Substituting (10) and (11) into (13), respectively, yields,

$$
\begin{aligned}
& \overline{P E P}_{\text {Approx. }}^{M R C}=\frac{K(N-K)}{2 \bar{\gamma}^{L}(L-1) !} \underbrace{\int_{0}^{\infty} \gamma^{L-1} e^{-\frac{\gamma(2+\bar{\gamma})}{2 \bar{\gamma}}} d \gamma}_{I_{3}} . \\
& \overline{P E P}^{M R C}=\frac{K}{\bar{\gamma}^{L}(L-1) !}[\underbrace{\int_{0}^{\infty} \gamma^{L-1} e^{-\gamma\left(\frac{1}{\bar{\gamma}}\right)} d \gamma}_{I_{1}}-\sum_{q=0}^{N-K} \\
& \times\left(\begin{array}{c}
N-K \\
q
\end{array}\right) \frac{(-1)^{q}}{q+1} \underbrace{\int_{0}^{\infty} \gamma^{L-1} e^{\gamma \frac{q \bar{\gamma}+q+1}{\bar{\gamma}(q+1)}} d \gamma}_{I_{2}}] \text {, }
\end{aligned}
$$

It can be seen from (A.2) and (A.1) that there are 3 integrals that are in the same form, i.e., $\int_{0}^{\infty} x^{\nu-1} e^{-\mu x} d x$. The integral solution can be found in [29, eq. (3.381-3], and follow the same form, i.e., $\mu^{-\nu} \Gamma(\nu)$. Therefore, $I_{1}=\bar{\gamma}^{L}(L-1)$ !, $I_{2}=$ $\left(\frac{\bar{\gamma}(q+1)}{q(\bar{\gamma}+1)+1}\right)^{L}(L-1) !$, and $I_{3}=\left(\frac{2 \bar{\gamma}}{\bar{\gamma}+2}\right)^{L}(L-1) !$

Substituting $I_{1}$ and $I_{2}$ into (A.2), and substituting $I_{3}$ in (A.1) yields the expressions (14) and (15), respectively.

\section{APPENDIX B}

DERIVATION OF (19) AND (20)

Substituting (10) and (11) into (18), respectively, yields,

$$
\begin{aligned}
\overline{P E P}{ }^{S C}= & \frac{K L}{\bar{\gamma}} \sum_{l=0}^{L-1}\left(\begin{array}{c}
L-1 \\
l
\end{array}\right)(-1)^{l}[\underbrace{\int_{0}^{\infty} e^{\gamma\left(-\frac{l+1}{\gamma}\right) d \gamma}}_{I_{4}} \\
& -\sum_{q=0}^{N-K}\left(\begin{array}{c}
N-K \\
q
\end{array}\right) \frac{(-1)^{q}}{q+1} \underbrace{\int_{0}^{\infty} e^{\gamma\left(\frac{1}{q+1}-1-\frac{l+1}{\gamma}\right) d \gamma}}_{I_{5}}],
\end{aligned}
$$




$$
\begin{aligned}
\overline{P E P}_{\text {Approx. }}^{S C} & =\frac{K L(N-K)}{2 \bar{\gamma}} \sum_{l=0}^{L-1}\left(\begin{array}{c}
L-1 \\
l
\end{array}\right)(-1)^{l} \\
& \times \underbrace{\int_{0}^{\infty} e^{\gamma\left(-\frac{l+1}{\bar{\gamma}}-\frac{1}{2}\right)} d \gamma}_{I_{6}}
\end{aligned}
$$

It can be seen from (B.1) and (B.2) that there are 3 integrals that are in the same form, i.e., $\int_{0}^{\infty} e^{-p x} d x$. The integral solution can be found in [29, eq. (3.310], and follow the same form, i.e., $1 / p$. Therefore, $I_{4}=\frac{\bar{\gamma}}{l+1}, I_{5}=\frac{\bar{\gamma}(q+1)}{l+q+l q+q \bar{\gamma}+1}$, and $I_{6}=\frac{2 \bar{\gamma}}{2 l+\bar{\gamma}+2}$.

Substituting the integral solutions for $I_{4}$ and $I_{5}$ into (B.1), and substituting the integral solution $I_{6}$ in (B.2) yields the expressions (19) and (20), respectively.

\section{APPENDIX C \\ DERIVATION OF (30) AND (31)}

Substituting (27) and (28) into (29), respectively, yields,

$$
\begin{aligned}
& \bar{P}_{s}^{M R C} \leq \frac{K}{\bar{\gamma}^{L}(L-1) !} \underbrace{\int_{0}^{\infty} \gamma^{L-1} e^{\gamma\left(-\frac{1}{\gamma}\right)} d \gamma}_{I_{7}} \\
& -K \sum_{q=0}^{N-K}\left(\begin{array}{c}
N-K \\
q
\end{array}\right) \frac{(-1)^{q}}{\bar{\gamma}^{L}(q+1)(L-1) !} \\
& \times \underbrace{\int_{0}^{\infty} \gamma^{L-1} e^{\gamma\left(\frac{1}{q+1}-1-\frac{1}{\gamma}\right)} d \gamma}_{I_{8}} \\
& +\frac{K}{\bar{\gamma}^{L}(L-1) !} \underbrace{\int_{0}^{\infty} \gamma^{L-1} e^{\gamma\left(-\frac{3}{2(M-1)}-\frac{1}{\gamma}\right)} d \gamma}_{I_{9}} \\
& -K \sum_{q=0}^{N-K}\left(\begin{array}{c}
N-K \\
q
\end{array}\right) \frac{(-1)^{q}}{(q+1) \bar{\gamma}^{L}(L-1) !} \\
& \underbrace{\int_{0}^{\infty} \gamma^{L-1} e^{\gamma\left(\frac{1}{q+1}-1-\frac{3}{2(M-1)}-\frac{1}{\gamma}\right)} d \gamma}_{I_{10}}, \\
& \bar{P}_{s, \text { Approx. }}^{M R C}=K \frac{N-K}{2 \bar{\gamma}^{L}(L-1) !} \underbrace{\int_{0}^{\infty} \gamma^{L-1} e^{\gamma\left(-\frac{1}{2}-\frac{1}{\gamma}\right)} d \gamma}_{I_{11}} \\
& -K \frac{N-K}{2 \bar{\gamma}^{L}(L-1) !} \\
& \times \underbrace{\int_{0}^{\infty} \gamma^{L-1} e^{\gamma\left(-\frac{1}{2}-\frac{3}{2(M-1)}-\frac{1}{\gamma}\right)} d \gamma}_{I_{12}} \\
& +\frac{1}{\bar{\gamma}^{L}(L-1) !} \underbrace{\int_{0}^{\infty} \gamma^{L-1} e^{\gamma\left(-\frac{3}{2(M-1)}-\frac{1}{\gamma}\right)} d \gamma}_{I_{13}} .
\end{aligned}
$$

Similar to the derivation of (14) and (15), the unknown integrals in the same form, i.e., $\int_{0}^{\infty} x^{v-1} e^{-\mu x} d x$, and therefore their solutions also follow the same form, i.e., $\mu^{-\nu} \Gamma(\nu)$. Where $\nu=L$ for $I_{7}$ to $I_{13}$, and $\mu=\left(\frac{1}{\bar{\gamma}}\right),\left(-\frac{1}{q+1}+1+\frac{1}{\bar{\gamma}}\right)$, $\left(\frac{3}{2(M-1)}+\frac{1}{\bar{\gamma}}\right), \quad\left(-\frac{1}{q+1}+1+\frac{3}{2(M-1)}+\frac{1}{\bar{\gamma}}\right), \quad\left(\frac{1}{2}+\frac{1}{\bar{\gamma}}\right)$, $\left(\frac{1}{2}+\frac{3}{2(M-1)}+\frac{1}{\bar{\gamma}}\right)$, and $\left(\frac{3}{2(M-1)}+\frac{1}{\bar{\gamma}}\right)$, for $I_{7}$ to $I_{13}$, respectively. Substituting the integral solutions for $I_{7}, I_{8}, I_{9}$, and $I_{10}$ into (C.1), and substituting the integral solutions for $I_{11}, I_{12}$, and $I_{13}$ into (C.2) yields the expressions (30) and (31), respectively. The solutions to the unknown integrals have been omitted in order to save space.

\section{APPENDIX D}

DERIVATION OF (37) AND (38)

Substituting (27) and (28) into (36), respectively, yields,

$$
\begin{aligned}
& \bar{P}_{s}^{S C} \leq K \frac{L}{\bar{\gamma}} \sum_{l=0}^{L-1}\left(\begin{array}{c}
L-1 \\
l
\end{array}\right)(-1)^{l} \underbrace{\int_{0}^{\infty} e^{\gamma\left(-\frac{l+1}{\gamma}\right)} d \gamma}_{I_{14}} \\
& -K \sum_{q=0}^{N-K}\left(\begin{array}{c}
N-K \\
q
\end{array}\right) \frac{(-1)^{q}}{q+1} \frac{L}{\bar{\gamma}} \sum_{l=0}^{L-1}\left(\begin{array}{c}
L-1 \\
l
\end{array}\right)(-1)^{l} \\
& \times \underbrace{\int_{0}^{\infty} e^{\gamma\left(\frac{1}{q+1}-1-\frac{l+1}{\gamma}\right)} d \gamma}_{I_{15}} \\
& +K \frac{L}{\bar{\gamma}} \sum_{l=0}^{L-1}\left(\begin{array}{c}
L-1 \\
l
\end{array}\right)(-1)^{l} \underbrace{\int_{0}^{\infty} e^{\gamma\left(-\frac{3}{2(M-1)}-\frac{l+1}{\gamma}\right)} d \gamma}_{I_{16}} \\
& -K \sum_{q=0}^{N-K}\left(\begin{array}{c}
N-K \\
q
\end{array}\right) \frac{(-1)^{q}}{q+1} \frac{L}{\bar{\gamma}} \sum_{l=0}^{L-1}\left(\begin{array}{c}
L-1 \\
l
\end{array}\right)(-1)^{l} \\
& \times \underbrace{\int_{0}^{\infty} e^{\gamma\left(\frac{1}{q+1}-1-\frac{3}{2(M-1)}-\frac{l+1}{\bar{\gamma}}\right)} d \gamma}_{I_{17}} \\
& \bar{P}_{s, \text { Approx. }}^{S C}=K \frac{N-K}{2} \frac{L}{\bar{\gamma}} \sum_{l=0}^{L-1}\left(\begin{array}{c}
L-1 \\
l
\end{array}\right)(-1)^{l} \\
& \underbrace{\int_{0}^{\infty} e^{\gamma\left(-\frac{1}{2}-\frac{l+1}{\bar{\gamma}}\right)} d \gamma}_{I_{18}}-K \frac{N-K}{2} \frac{L}{\bar{\gamma}} \\
& \times \sum_{l=0}^{L-1}\left(\begin{array}{c}
L-1 \\
l
\end{array}\right)(-1)^{l} \underbrace{\int_{0}^{\infty} e^{\gamma\left(-\frac{1}{2}-\frac{3}{2(M-1)}-\frac{l+1}{\bar{\gamma}}\right)} d \gamma}_{I_{19}} \\
& +\frac{L}{\bar{\gamma}} \sum_{l=0}^{L-1}\left(\begin{array}{c}
L-1 \\
l
\end{array}\right)(-1)^{l} \underbrace{\int_{0}^{\infty} e^{\gamma\left(-\frac{3}{2(M-1)}-\frac{l+1}{\gamma}\right)} d \gamma}_{I_{20}} .
\end{aligned}
$$

Similar to the derivation of (19) and (20), the unknown integrals in the same form, i.e., $\int_{0}^{\infty} e^{-p x} d x$. The integral solution can be found in [29, eq. (3.310], and follow the same form, i.e., $1 / p$. Where $p=\left(\frac{l+1}{\bar{\gamma}}\right),\left(-\frac{1}{q+1}+1+\frac{l+1}{\bar{\gamma}}\right)$, $\left(\frac{3}{2(M-1)}+\frac{l+1}{\bar{\gamma}}\right), \quad\left(-\frac{1}{q+1}+1+\frac{3}{2(M-1)}+\frac{l+1}{\bar{\gamma}}\right)$, $\left(\frac{1}{2}+\frac{l+1}{\bar{\gamma}}\right),\left(\frac{1}{2}+\frac{3}{2(M-1)}+\frac{l+1}{\bar{\gamma}}\right)$, and $\left(\frac{3}{2(M-1)}+\frac{l+1}{\bar{\gamma}}\right)$, 
for $I_{14}$ to $I_{20}$, respectively. Substituting the integral solutions for $I_{14}, I_{15}, I_{16}$, and $I_{17}$ into (D.1), and substituting the integral solutions for $I_{18}, I_{19}$, and $I_{20}$ into (D.2) yields the expressions (37) and (38), respectively. The solutions to the unknown integrals have been omitted in order to save space.

\section{ACKNOWLEDGMENT}

The research leading to the results in this work has been supported by the Engineering and Physical Sciences Research Council under grant reference number EP-M015521-1.

\section{REFERENCES}

[1] R. Abu-alhiga and H. Haas, "Subcarrier-index modulation OFDM," IEEE Int. Sym. Personal, Indoor and Mobile Radio Commun., pp. 177181, Sep. 2009.

[2] D. Tsonev, S. Sinanovic, and H. Haas, "Enhanced subcarrier index modulation (SIM) OFDM," Proc. IEEE GLOBECOM Workshops, pp. 728-732, Dec. 2011.

[3] R. Y. Mesleh, H. Haas, S. Sinanovic, C. W. Ahn, and S. Yun, "Spatial modulation," IEEE Trans. Veh. Technol., vol. 57, no. 4, pp. 2228-2241, Jul. 2008 .

[4] A. Younis, N. Serafimovski, R. Y. Mesleh, and H. Haas, "Generalised spatial modulation," Proc. Asilomar Conf. Signals, Syst., Comput., pp. 1498-1502, Nov. 2010.

[5] G. Kaddoum, M. F. A. Ahmed, and Y. Nijsure, "Code index modulation: A high data rate and energy efficient communication system," IEEE Commun. Lett., vol. 19, no. 2, pp. 175-178, Feb. 2015.

[6] G. Kaddoum, Y. Nijsure, and H. Tran, "Generalized code index modulation technique for high-data-rate communication systems," IEEE Trans. Veh. Technol., vol. 65, no. 9, pp. 7000-7009, Sept. 2016.

[7] G. Kaddoum and E. Soujeri, "On the comparison between code-index modulation and spatial modulation techniques," Int. Conf. Inform. and Commun. Technol. Research, pp. 24-27, Jul. 2015.

[8] P. Yang, Y. Xiao, Y. L. Guan, K. V. S. Hari, A. Chockalingam, S. Sugiura, H. Haas, M. Di Renzo, C. Masouros, Z. Liu, L. Xiao, S. Li, and L. Hanzo, "Single-carrier sm-mimo: A promising design for broadband large-scale antenna systems," IEEE Commun. Surveys Tuts., vol. 18, no. 3, pp. 1687-1716, 3rd Quart. 2016.

[9] E. Basar, U. Aygolu, E. Panayirci, and H. V. Poor, "Orthogonal frequency division multiplexing with index modulation," IEEE Trans. on Signal Process., vol. 61, no. 22, pp. 5536-5549, Nov. 2013.

[10] Y. Ko, "A Tight Upper Bound on Bit Error Rate of Joint OFDM and Multi-Carrier Index Keying," IEEE Commun. Lett., vol. 18, no. 10, pp. 1763-1766, Oct. 2014.

[11] Y. Xiao, S. Wang, L. Dan, X. Lei, P. Yang, and W. Xiang, "OFDM with interleaved subcarrier-index modulation," IEEE Commun. Lett., vol. 18, no. 8, pp. 1447-1450, Aug. 2014.

[12] X. Cheng, L. Wen, L. Yang, and Y. Li, "Index modulated OFDM with interleaved grouping for V2X communications," IEEE Int. Conf. Intelligent Transportation Systems, pp. 1097-1104, Oct. 2014.

[13] S. Hong, M. Sagong, C. Lim, S. Cho, K. Cheun, and K. Yang, "Frequency and quadrature-amplitude modulation for downlink cellular OFDMA networks," IEEE Journal on Selected Areas in Communications, vol. 2014, no. 6, pp. 1256-1267, Jun. 2014.

[14] M. Wen, X. Cheng, and L. Yang, "Optimizing the energy efficiency of OFDM with index modulation," IEEE Int. Conf. Commun. Workshops, pp. 31-35, Nov. 2014.

[15] W. Li, H. Zhao, C. Zhang, L. Zhao, and R. Wang, "Generalized selecting sub-carrier modulation scheme in OFDM systems," IEEE Int. Conf. Commun. Workshops, pp. 907-911, Jun. 2014.

[16] E. Basar, "OFDM with index modulation using coordinate interleaving," IEEE Wireless Commun. Lett., vol. 4, no. 4, pp. 381-384, Aug. 2015.

[17] R. Fan, Y. Yu, and Y. L. Guan, "Generalization of orthogonal frequency division multiplexing with index modulation," IEEE Transactions on Wireless Commun., vol. 14, no. 10, pp. 5350-5359, Oct. 2015.

[18] J. Choi and Y. Ko, "Compressive sensing based detector for sparse signal modulation in precoded OFDM," Proc. IEEE Int. Conf. on Commun., pp. 4536-4540, Jun. 2015.

[19] Y. Ko and J. Choi, "Sparse multi-carrier index keying OFDM with index separation over correlated sub-carriers," Int. Conf. Inform. and Commun. Technology Convergence, pp. 307-312, Oct. 2015
[20] M. Wen, X. Cheng, M. Ma, B. Jiao, and H. V. Poor, "On the achievable rate of OFDM with index modulation," IEEE Trans. Signal Process. vol. 64, no. 8, pp. 1919-1932, Apr. 2016.

[21] A. I. Siddiq, "Low complexity OFDM-IM detector by encoding all possible subcarrier activation patterns," IEEE Commun. Lett., vol. 20, no. 3, pp. 446-449, Mar. 2016.

[22] B. Zheng, F. Chen, M. Wen, F. Ji, H. Yu, and Y. Liu, "Lowcomplexity ML detector and performance analysis for OFDM with inphase/quadrature index modulation," IEEE Commun. Lett., vol. 19, pp. 1893-1896, Nov. 2015.

[23] E. Basar, "Multiple-input multiple-Output OFDM with index modulation," IEEE Signal Process. Lett., vol. 22, no. 12, pp. 2259-2263, Dec. 2015.

[24] J. Crawford and Y. Ko, "Low Complexity Greedy Detection Method with Generalized Multicarrier Index Keying OFDM," Proc. IEEE Pers., Indoor., Mobile Radio Commun., pp. 688-693, Aug. 2015.

[25] E. Chatziantoniou, J. Crawford, and Y. Ko, "Performance analysis of a low complexity detector for MCIK-OFDM over TWDP fading," IEEE Commun. Lett., vol. 20, pp. 1251-1254, Jun. 2016.

[26] —, "A low complexity detector with MRC diversity reception for MCIK-OFDM," Proc. IEEE Pers., Indoor., Mobile Radio Commun., Dec. 2016.

[27] A. Goldsmith, Wireless communications. Cambridge University Press, 2005.

[28] E. Basar, "On multiple-input multiple-Output OFDM with index modulation for next generation wireless networks," IEEE Trans. Signal Process., vol. 64, no. 15, pp. 3868-3878, Aug. 2016.

[29] I. Gradshteyn and I. Ryzhik, Table of Integrals, Series, and Products, A. Jeffrey and D. Zwillinger, Eds. Academic Press, 2007.

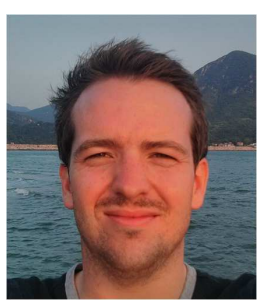

James Crawford James Crawford received the M.Eng. degree in electrical and electronic engineering from Queen's University, Belfast, Northern Ireland, in 2014. He is currently pursuing the $\mathrm{Ph} . \mathrm{D}$. degree with Queen's University Belfast, Northern Ireland. His research is in the areas of multicarrier index keying, and low power device type communications, for the next generation wireless communications.

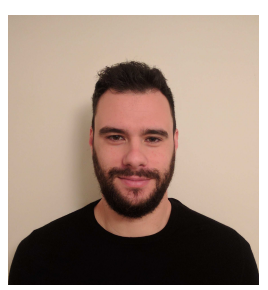

Eleftherios Chatziantoniou received the B.Sc. degree in Computer Engineering from Piraeus University of Applied Sciences, Greece in 2009. He subsequently received the M.Sc. degree in Communications and Signal Processing from University of Bristol, U.K. in 2010 and the Ph.d. degree in Wireless Communications from the University of Bedfordshire, U.K. in 2015. From 2014 to 2015, he was with Sepura as an R\&D Engineer. In 2015 he joined the Centre for Wireless Innovation, Queen's University Belfast, U.K. as a Research Fellow. His research interests lie within the areas of signal processing, cognitive radio, wireless device-to-device communications, multi-carrier index keying, and performance analysis of fading channels.

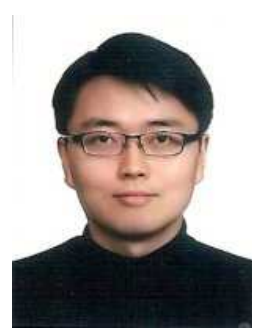

Youngwook Ko Dr. Ko received Ph.D. in 2006 and M.S. in 2002, in Electrical Engineering from Arizona State University, Tempe, AZ USA, and B.S.E. in Information and Communications Engineering from Hannam University, South Korea. After his Ph.D., he worked at Samsung for two years, as a senior researcher. In 2008, he was in Electrical and Computer Engineering at the University of Alberta, Canada. Between 2010-2013 he was with the CCSR, University of Surrey, UK, as a senior research fellow. Since 2013, Dr. Ko has been with the ECIT Institute at the Queen's University of Belfast as a Lecturer in Wireless Communications Innovation Centre. He has authored over 30 publications in major IEEE international journals, and peer-reviewed international conferences. Dr Ko is the recipient of several EPSRC and Newton projects such as the EPSRC First Grant Award and the EPSRC DTP. He is on the Editorial Board of the Elsevier Journal on Physical Communications and a member of the EPSRC Peer Review Associate College. His research is in the areas of multicarrier index keying, machine type communications, wireless security, industrial wireless communications and machine type multiple access, for the next generation wireless device-centric communications. 